\title{
MERTK inhibition alters the PD-1 axis and promotes anti-leukemia immunity
}

\author{
Alisa B. Lee-Sherick, ${ }^{1}$ Kristen M. Jacobsen, ${ }^{2,3,4}$ Curtis J. Henry, ${ }^{3,4}$ Madeline G. Huey, ${ }^{3,4}$ \\ Rebecca E. Parker, ${ }^{3,4}$ Lauren S. Page, ${ }^{1}$ Amanda A. Hill, ${ }^{1}$ Xiaodong Wang, ${ }^{5}$ Stephen V. Frye,, \\ H. Shelton Earp, ${ }^{6,7}$ Craig T. Jordan, ${ }^{8}$ Deborah DeRyckere, ${ }^{3,4}$ and Douglas K. Graham ${ }^{3,4}$ \\ 'Department of Pediatrics, ${ }^{2}$ Department of Immunology and Microbiology, University of Colorado Anschutz Medical \\ Campus, Aurora, Colorado, USA. ${ }^{3}$ Department of Pediatrics, Emory University, Atlanta, Georgia, USA. ${ }^{4}$ Aflac Cancer and \\ Blood Disorders Center, Children's Healthcare of Atlanta, Atlanta, Ceorgia, USA. ${ }^{5}$ Center for Integrative Chemical Biology \\ and Drug Discovery, Division of Chemical Biology and Medicinal Chemistry, Eshelman School of Pharmacy, ${ }^{6}$ Lineberger \\ Comprehensive Cancer Center, and 'Departments of Medicine and Pharmacology, University of North Carolina School of \\ Medicine, Chapel Hill, North Carolina, USA. ${ }^{8}$ Division of Hematology, University of Colorado, Aurora, Colorado, USA.
}

MERTK is ectopically expressed and promotes survival in acute lymphoblastic leukemia (ALL) cells and is thus a potential therapeutic target. Here we demonstrate both direct therapeutic effects of MERTK inhibition on leukemia cells and induction of anti-leukemia immunity via suppression of the coinhibitory PD-1 axis. A MERTK-selective tyrosine kinase inhibitor, MRX-2843, mediated therapeutic anti-leukemia effects in immunocompromised mice bearing a MERTK-expressing human leukemia xenograft. In addition, inhibition of host MERTK by genetic deletion (Mertk ${ }^{-1-}$ mice) or treatment with MRX-2843 significantly decreased tumor burden and prolonged survival in immune-competent mice inoculated with a MERTK-negative ALL, suggesting immune-mediated therapeutic activity. In this context, MERTK inhibition led to significant decreases in expression of the coinhibitory ligands PD-L1 and PD-L2 on CD11b+ monocytes/macrophages in the leukemia microenvironment. Furthermore, although T cells do not express MERTK, inhibition of MERTK indirectly decreased PD- 1 expression on $\mathrm{CD}^{+}$and $\mathrm{CD} 8^{+} \mathrm{T}$ cells and decreased the incidence of splenic FOXP3 $^{+}$Tregs at sites of leukemic infiltration, leading to increased T cell activation. These data demonstrate direct and immune-mediated therapeutic activities in response to MERTK inhibition in ALL models and provide validation of a translational agent targeting MERTK for modulation of tumor immunity.

Authorship note: ABLS and KMJ contributed equally to this work.

Conflict of interest: SVF and XW are inventors on a patent describing MRX-2843 (W02013052417). DKG, HSE, SVF, XW, and DDR are stockholders in Meryx Inc.

License: Copyright 2018, American Society for Clinical Investigation.

Submitted: October 9, 2017 Accepted: September १९, 2018 Published: November 2, 2018

Reference information: JCI Insight. 2018;3(21):e97941. https://doi.org/10.1172/jici. insight.97941.

\section{Introduction}

Many patients with acute lymphoblastic leukemia (ALL), particularly pediatric patients, are cured by treatment with standard cytotoxic chemotherapies; however, for patients with refractory or relapsed leukemia, there is significant need for new therapies that eliminate disease, ideally with fewer toxic side effects. New treatments are also critically needed for elderly patients, who often cannot tolerate standard chemotherapy, and pediatric patients, who may experience lifelong side effects of current therapies. Molecularly targeted agents present an attractive option to specifically and effectively eliminate leukemia cells with potential for limited toxic side effects. One potential target in ALL is the MERTK receptor tyrosine kinase.

MERTK is ectopically expressed in approximately $30 \%$ of pediatric B cell ALL (B-ALL) (1) and 40\%$50 \%$ of pediatric T cell ALL (2) patient samples and is not expressed on lymphocytes under physiologic conditions. In ALL cells, MERTK activates intracellular signal transduction through proliferative and pro-survival pathways such as AKT and ERK1/2 (1). Additionally, MERTK activation is associated with induction of an antiapoptotic gene expression signature in B-ALL cells (1). Specifically, when MERTK was inhibited using RNA interference (shRNA), antiapoptotic genes BCL2L1 (BCL-XL), PIK3R5 (PI3K), and PRKCB (PKC) were downregulated and proapoptotic BAX, PMAIP1 (NOXA), and BBC3 (PUMA) were upregulated. This transcriptional program was accompanied by significant induction of apoptosis under stress conditions, decreased colony-forming potential and increased chemosensitivity in cell culture assays, and prolonged survival in xenograft models $(1,3)$. These data demonstrate direct antitumor activity mediated by MERTK inhibition and validate MERTK as a potential therapeutic target in ALL. 
The best-described physiologic role for MERTK is in efferocytosis, the phagocytic process by which macrophages and certain epithelial cells ingest apoptotic material (4-8). During efferocytosis, MERTK activation promotes polarization of macrophages toward an M2 phenotype and leads to immune tolerization of dendritic cells $(9,10)$. Mertk loss-of-function mutations in animal models have been associated with development of autoimmune diseases, such as systemic lupus erythematosus, further confirming a role in immune tolerance $(6,11,12)$. Recent data also implicate MERTK in antitumor immunity. In solid tumor models, mice with genetic deletion of Mertk had significantly reduced tumor burden and decreased incidence of metastases relative to WT controls $(13,14)$. These effects were recapitulated in mice transplanted with Mertk $^{-/}$bone marrow, implicating Mertk deletion in the hematopoietic compartment as a mechanism of antitumor activity (13). Decreased tumor growth was accompanied by proinflammatory cytokine production and mediated by $\mathrm{CD}^{+} \mathrm{T}$ cells. Additional studies suggest a role for MERTK in regulation of immune checkpoint signaling through CD274 (PD-L1) and programmed cell death 1 ligand 2 (PD-L2) (15, 16). PD-L1 and PD-L2 bind the programmed cell death 1 (PD-1) receptor on tumor-infiltrating $\mathrm{T}$ cells, which inhibits activation and promotes apoptosis of tumor-reactive $\mathrm{T}$ cells, preventing tumor rejection (17, 18). Expression of PD-1 or PD-L1 and PD-L2 is a prognostic factor in several types of cancer (19-23). In epithelial cells, expression of constitutively activated MERTK led to enhanced expression of PD-L1 and PD-L2 $(15,16)$, and shRNA-mediated inhibition of MERTK in a breast cancer cell line decreased PD-L1 expression (15). These studies indicate multiple mechanisms by which MERTK can contribute to immune suppression in the tumor microenvironment.

The demonstrated roles for MERTK in promoting both tumor cell survival and an immunosuppressive microenvironment that restricts antitumor immunity support a dual mechanism of action for MERTK-directed therapy. Thus, MERTK inhibition may provide a unique opportunity to directly impact tumor cell survival and promote immune-mediated tumor rejection by inhibition of a single target. To investigate this idea we utilized immune-competent mice that harbored a homozygous MERTK-knockout mutation (Mertk ${ }^{--}$) and treatment of WT mice with a small molecule MERTK tyrosine kinase inhibitor, MRX2843, in syngeneic models of ALL. MRX-2843 is a potent and selective small molecule MERTK and Fmslike tyrosine kinase 3 (FLT3) tyrosine kinase inhibitor that is currently in clinical development $(24,25)$. MRX-2843 and UNC2025, a compound with similar chemical structure that was developed earlier, mediate therapeutic activity in approximately $30 \%$ of leukemia patient samples and in numerous cell line and patient-derived xenograft models, both alone and in combination with cytotoxic chemotherapy, mimicking the effect of MERTK shRNA in xenograft models $(3,24,25)$. Thus, these compounds mediate robust anti-leukemia activity by direct inhibition of MERTK on leukemic blasts. Here we utilized both genetic and pharmacologic methods to inhibit MERTK function in the leukemia microenvironment to investigate whether inhibition of MERTK promotes immune-mediated clearance of ALL.

\section{Results}

$M R X-2843$ inhibits phosphorylation of MERTK in B-ALL cells in vitro and in vivo. MERTK phosphorylation was assessed in 697 B-ALL cells following treatment with the MERTK small molecule inhibitor MRX2843. Treatment with MRX-2843 in vitro resulted in decreased MERTK phosphorylation at doses as low as 5-25 nM, with near complete inhibition of detectable MERTK phosphoprotein at doses greater than $250 \mathrm{nM}$ (Supplemental Figure 1A; supplemental material available online with this article; https://doi. org/10.1172/jci.insight.97941DS1). To evaluate inhibition of MERTK mediated by MRX-2843 in vivo, 697 xenografts were generated, and mice with advanced leukemia were treated with a single oral dose (3, 25 , or $75 \mathrm{mg} / \mathrm{kg}$ ) of MRX-2843 or vehicle. Bone marrow was harvested 1 or 24 hours after treatment, and phosphorylation of human MERTK was assessed. MERTK was effectively dephosphorylated 1 hour after treatment with $3 \mathrm{mg} / \mathrm{kg}$ MRX-2843 (Supplemental Figure 1, B and C); however, at this dose, inhibition was not sustained over 24 hours (Supplemental Figure 1E). In contrast, MERTK phosphorylation was decreased by more than $90 \%$ after 24 hours in mice treated with $75 \mathrm{mg} / \mathrm{kg} \mathrm{MRX}-2843$. Thus, to attain continuous inhibition of MERTK with once-daily dosing, MRX-2843 was administered at $60-75 \mathrm{mg} / \mathrm{kg}$ in subsequent studies. A lower dose of $50 \mathrm{mg} / \mathrm{kg}$ was used in some studies to assess dose dependence.

$M R X-2843$ promotes survival in an immunocompromised murine B-ALL xenograft model. We previously reported robust therapeutic effects in immunocompromised models following MERTK inhibition in ALL cells using RNA interference (26) or UNC2025 (3). To investigate the efficacy of MRX-2843 in ALL models, xenografts were generated in NSG mice using a luciferase-expressing derivative of the 697 cell line, and 
A<smiles>[CH]=[CH]</smiles>

$697^{\text {luct }}$

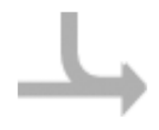

Start MRX-2843

one day after

transplantation
E

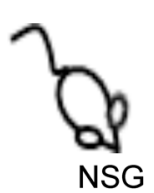

$697^{\text {luct }}$

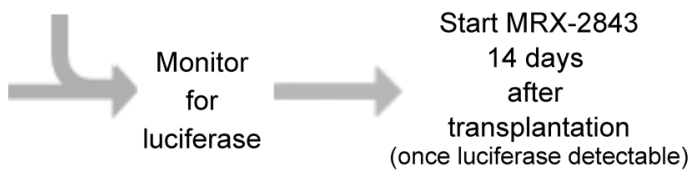

B

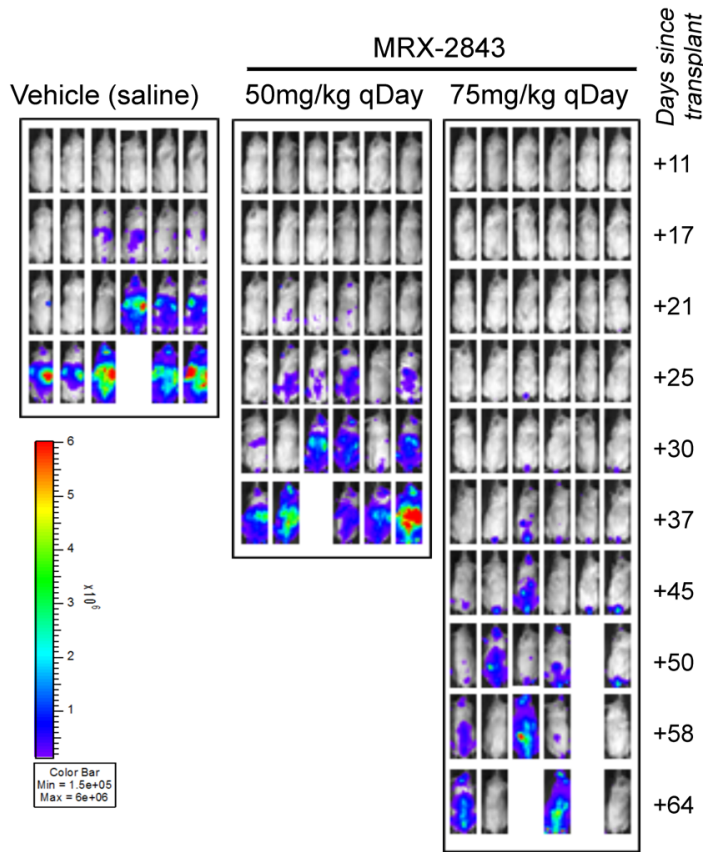

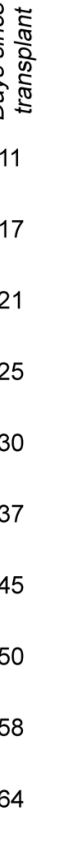

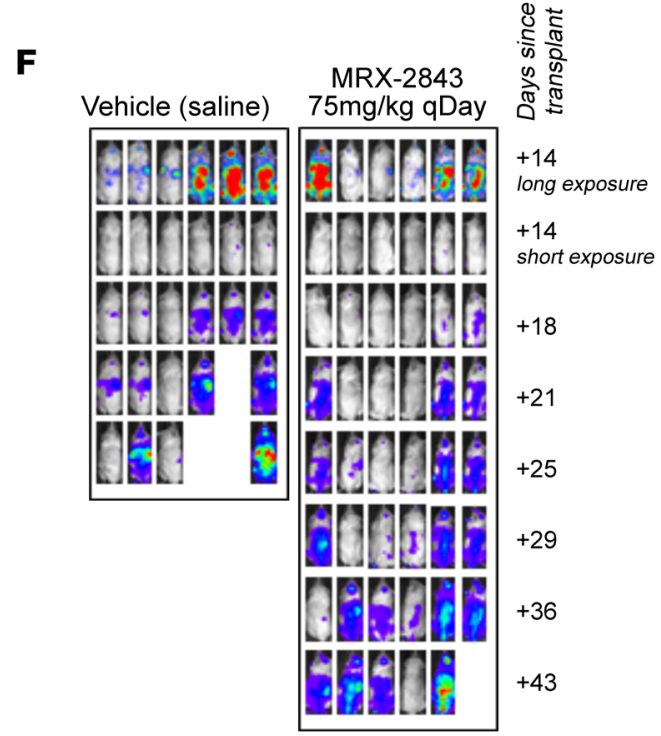

G
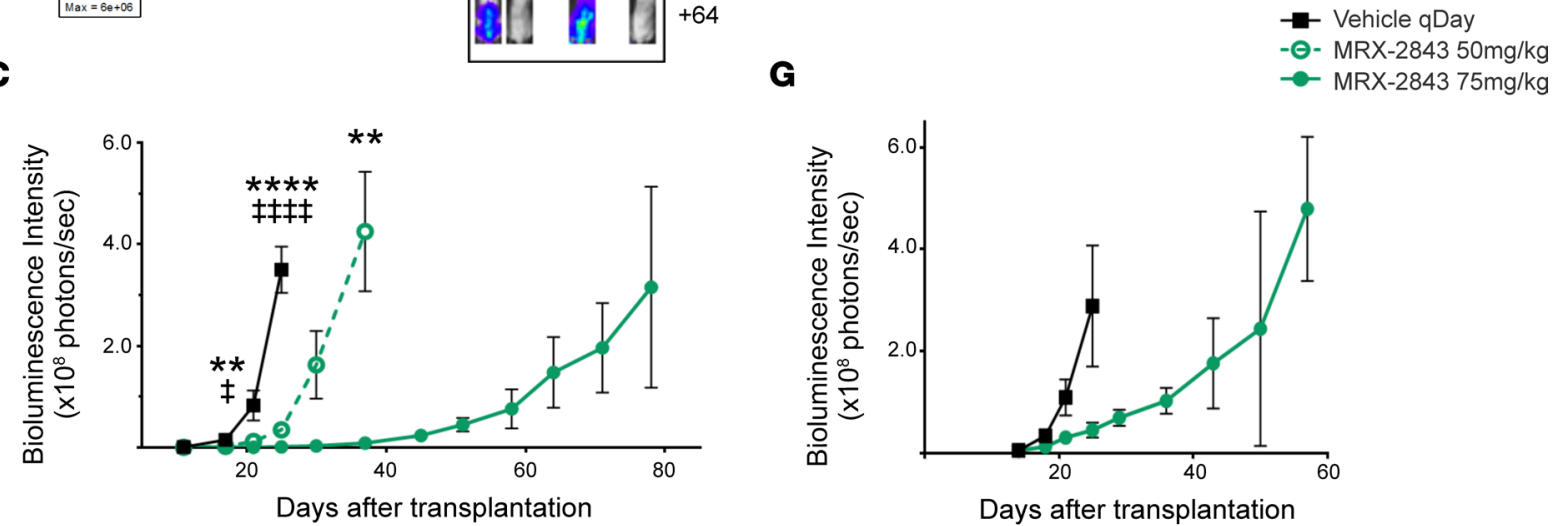

H

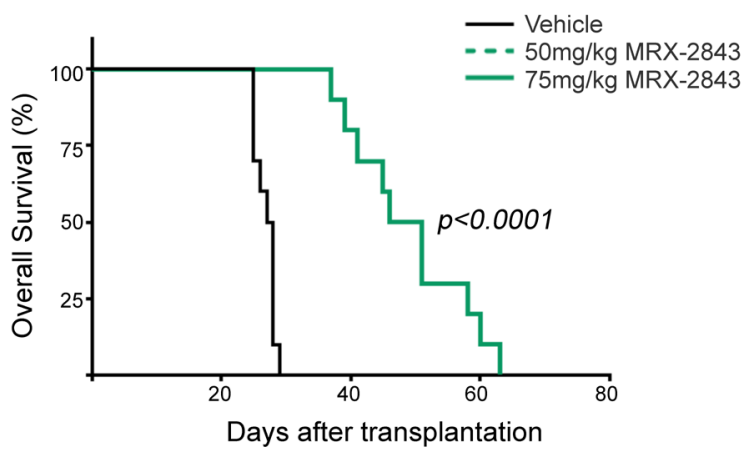

Figure 1. MERTK inhibitor MRX-2843 decreases leukemic burden and increases survival in an orthotopic ALL xenograft model. 697 B-ALL cells expressing the firefly luciferase gene were inoculated into NSG mice by tail vein injection. Disease burden was assessed by bioluminescence imaging, and survival was monitored. (A-D) Mice were treated with $50 \mathrm{mg} / \mathrm{kg}$ (dashed green line) or $75 \mathrm{mg} / \mathrm{kg}$ (solid green line) MERTK inhibitor MRX-2843 or an equivalent volume of vehicle (saline; solid black line) once daily, beginning 1 day after leukemia cell injection to model low leukemic burden. (B) Representative bioluminescence images. (C) Mean bioluminescence intensities and SEM $\left(n=10-11,{ }^{*} P<0.05,{ }^{* *} P<0.01,{ }^{* * * *} P<0.0001,{ }^{\ddagger} P<0.05,{ }^{, * \neq \pm} P<0.0001 ; 1\right.$-way ANOVA; ${ }^{*}$ comparison between saline and $75 \mathrm{mg} / \mathrm{kg}$ UNC2025); ${ }^{\ddagger}$ comparison between saline and $50 \mathrm{mg} / \mathrm{kg}$ UNC2025. (D) Kaplan-Meier survival curves ( $n=7-10, P<0.0001$ 
compared with vehicle, log-rank test). (E-H) Leukemic mice were randomized to groups with equal mean bioluminescence intensities and treated with $75 \mathrm{mg} / \mathrm{kg}$ MRX-2843 or an equivalent volume of vehicle (saline) daily, beginning 12 days after tumor inoculation to model existent disease. (F) Representative bioluminescence images. (G) Mean bioluminescence intensities and standard errors ( $n=10)$. (H) Kaplan-Meier survival curves ( $n=10, P<0.0001$ compared with vehicle, log-rank test). Data shown are representative of 2 independent experiments.

MRX-2843 was administered using two different approaches. First, treatment with MRX-2843 or vehicle was initiated 1 day after tumor inoculation, when disease burden was minimal (Figure 1A). In this model, MRX-2843 mediated a dose-dependent reduction in tumor burden indicated by decreased bioluminescence compared with vehicle treatment (Figure 1, B and C). Additionally, there was a dose-dependent increase in median survival from 27 days after inoculation with tumor cells in vehicle-treated mice to 39 and 80 days in mice treated with $50 \mathrm{mg} / \mathrm{kg}$ or $75 \mathrm{mg} / \mathrm{kg}$ MRX-2843, respectively $(P<0.0001$; Figure 1D). Extension of survival with more complete MERTK inhibition $(75 \mathrm{mg} / \mathrm{kg}$ ) was similar to previous studies using shRNA to decrease MERTK protein levels in 697 cells, providing strong evidence that MRX-2843 therapeutic activity was due to inhibition of MERTK (1). In a model of existent disease, leukemia was confirmed using bioluminescence imaging, and mice were randomized to groups with equal starting disease burden (data not shown) prior to initiation of therapy (Figure 1, E and F). In this model, treatment with MRX-2843 also mediated a reduction in tumor burden (Figure 1, F and G) and prolonged survival from 28 days after transplant in vehicle-treated mice to 49 days in mice treated with $75 \mathrm{mg} / \mathrm{kg} \mathrm{MRX}-2843(P<0.0001$; Figure $2 \mathrm{H})$. These data demonstrate direct antitumor activity mediated by MRX-2843 in immunocompromised mouse models of ALL and demonstrate therapeutic utility of MRX-2843 in the settings of both high and low disease burden.

MERTK inhibition has minimal effects on immune progenitors. Given the known roles for MERTK in the immune system and in the tumor microenvironment, we next sought to extend our findings by evaluating the impact of MERTK inhibition in immunocompetent models of ALL. To determine whether treatment with MRX-2843 alters immune cell development, we evaluated the impact of treatment with MRX-2843 on hematopoiesis and immune progenitors. In initial studies, we confirmed inhibition of murine MERTK mediated by MRX-2843 at concentrations similar to those required for inhibition of human MERTK in vitro (Supplemental Figure 2A). C57BL/6 mice were treated with $60 \mathrm{mg} / \mathrm{kg}$ MRX-2843 or an equivalent volume of vehicle for 24 days, and then bone marrow and peripheral blood were harvested. Complete blood counts were determined and revealed a slight but statistically insignificant decrease in white blood cell number that was not specific to any mature cell fraction (Supplemental Figure 2B). Although statistically significant changes in hemoglobin, red blood cell count, hematocrit, and red cell distribution width were noted, these differences are unlikely to have clinical relevance, given their minimal magnitude of change (Supplemental Figure 2C). Hypochromia was not observed. Platelet number and volume were unaffected in MRX-2843-treated mice (Supplemental Figure 2D). Although peripheral blood counts were relatively normal, mean bone marrow cellularity was significantly decreased from $34.8 \times 10^{6}$ cells in vehicle-treated mice to $19.6 \times 10^{6}$ cells in mice treated with MRX-2843 $(P<0.001$; Supplemental Figure $2 \mathrm{E})$. To further characterize the changes in bone marrow, hematopoietic stem cells (HSCs) and myeloid progenitors were quantitated by flow cytometry. There was a significant increase in the number of short-term HSCs from $0.37 \times 10^{5}$ in vehicle-treated mice to $0.64 \times 10^{5}$ in mice treated with $60 \mathrm{mg} / \mathrm{kg} \mathrm{MRX}-2843(P<0.05$; Supplemental Figure 3, A and B). No significant differences were observed in other lineage-negative progenitor populations, including long-term HSCs and multipotent progenitors. In more committed progenitors, there was a significant decrease in the megakaryocyte/erythroid progenitor (MEP) population from $1.29 \times 10^{5}$ cells in vehicle-treated mice to $0.33 \times 10^{5}$ in bone marrow from mice treated with $60 \mathrm{mg} / \mathrm{kg} \mathrm{MRX}-2843$ ( $P$ $<0.001$; Supplemental Figure 3, C and D), although this did not result in substantial anemia or thrombocytopenia (Supplemental Figure 2, C and D).

MERTK inhibition mediates immunotherapeutic effects in a MERTK-negative B-ALL model. To evaluate potential immune-mediated effects of MERTK inhibition, mice that harbored a homozygous MERTK-knockout mutation (Mertk $\left.{ }^{-/}\right)$(27) and WT C57BL/6J mice were inoculated with a syngeneic murine leukemia cell line that contains an $A r f$-null mutation and expresses the p185 variant of the $B C R-A B L$ oncogene (Arf/p $\left.185^{+}\right)(28,29)$. This B-ALL cell line also expresses GFP, but does not express MERTK (Figure 2A) or FLT-3 (Supplemental Figure 4A), such that any effects of MRX-2843 would be independent of direct effects on leukemia cells. Similarly, little or no MERTK expression was detected on $\mathrm{Arf}^{\prime-}$ p185 B-ALL cells in vivo (Supplemental Figure 4B). Median survival of WT mice was 21 days, whereas all but one Mertk ${ }^{-1}$ mouse were still alive 150 days after transplant $(P<0.0001$; Figure $2 \mathrm{~B})$. Clinical symptoms of leukemia 
$\mathbf{A}$

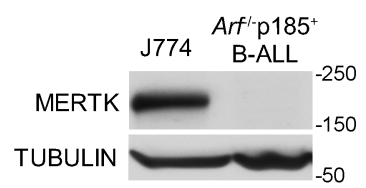

B

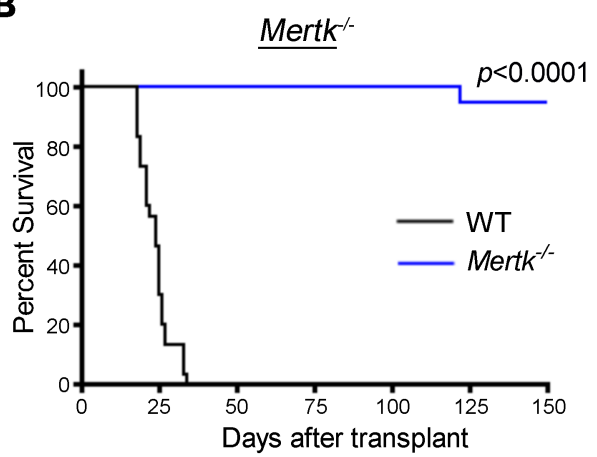

D

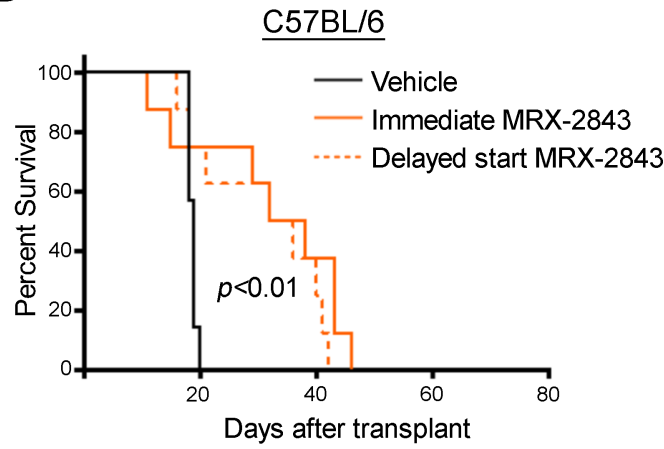

C

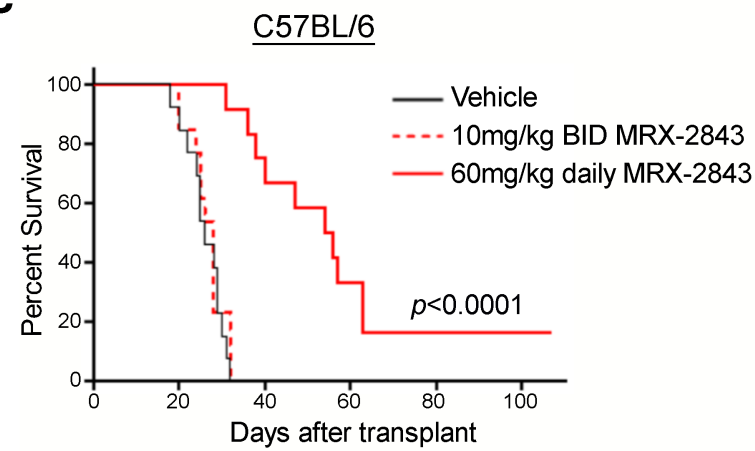

E

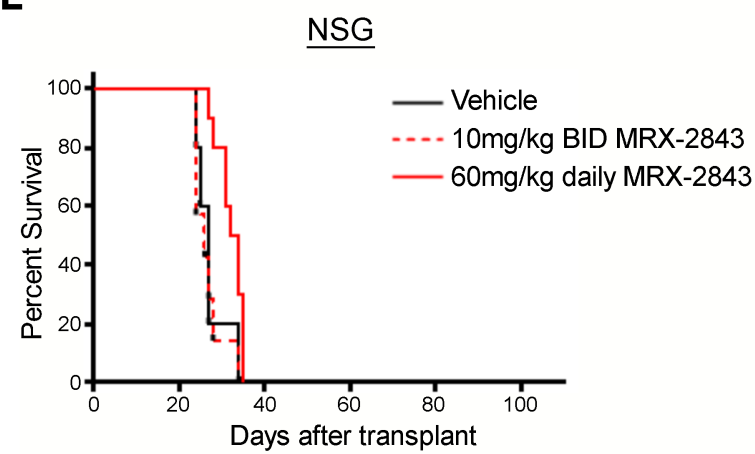

Figure 2. MERTK inhibition increases survival in an immunocompetent MERTK-negative B-ALL model. WT or Mertk 1 - C57BL/6 mice were inoculated with $5 \times 10^{4} \mathrm{GFP}$-expressing $\mathrm{Arf}^{-/-}$p $185^{+} \mathrm{B}$-ALL cells by tail vein injection. (A) MERTK expression was not detected in the Arf ${ }^{-1-} B C R-A B L \mathrm{p} 185^{+} \mathrm{B}-\mathrm{ALL}$ cell line by immunoblot. The $\mathbf{7 7 4}$ murine macrophage-like cell line is shown as a positive control. (B) Kaplan-Meier survival curves derived from WT or Mertk-1 C57BL/6 mice injected with B-ALL cells (WT $n=30$ and Mertk $^{-1-} n=20$ in 3 independent cohorts). (C) Kaplan-Meier survival curves derived from WT mice injected with B-ALL cells and treated with $10 \mathrm{mg} / \mathrm{kg}$ MERTK inhibitor MRX-2843 twice daily (BID), $60 \mathrm{mg} / \mathrm{kg}$ MRX-2843 once daily, or an equivalent volume of vehicle (saline) beginning 1 day after transplant ( $n=13$ in 2 independent cohorts). (D) Kaplan-Meier survival curve derived from WT mice injected with B-ALL cells and treated with $60 \mathrm{mg} / \mathrm{kg}$ MRX-2843 once daily starting the day after injection (Immediate MRX-2843) or 5 days after injection (Delayed start MRX-2843), or an equivalent volume of vehicle (saline) beginning 1 day after transplant ( $n=8$, representative of 2 independent cohorts). (E) Kaplan-Meier survival curve derived from NSG mice injected with 5,000 $\mathrm{Arf}^{-/-}$p $185^{+}$cells $(n=6-10$, representative of 2 independent experiments). Statistically significant differences were determined compared with WT or vehicle using a log-rank test.

were not observed in any of the $\mathrm{Mertk}^{-/-}$mice during the study, and leukemia was not confirmed in the single $\mathrm{Mertk}^{-/-}$mouse that died. To assess the effects of pharmacologic MERTK inhibition, WT C57BL/6J mice were inoculated with $A f^{\prime-}$ p185 cells, and treatment with MRX-2843 was initiated 1 day later. Median survival was significantly prolonged in mice treated with $60 \mathrm{mg} / \mathrm{kg}$ MRX-2843 daily (55 days) compared with vehicle-treated mice ( 26 days, $P<0.0001$; Figure $2 \mathrm{C}$ ). In contrast, a lower dose of $10 \mathrm{mg} / \mathrm{kg}$ administered twice daily had no significant impact on survival (28 days; $P>0.50$ ). In a subsequent study, median survival was similarly prolonged in mice treated with MRX-2843 compared with vehicle-treated mice (20 days), irrespective of whether treatment was initiated 1 day (38 days, $P<0.01$ ) or 5 days ( 40 days, $P<0.01$ ) after B-ALL inoculation (Figure 2D). In contrast, there was no significant difference in median survival in immunocompromised NSG mice inoculated with $\mathrm{Arf}^{\prime-} \mathrm{p} 185^{+}$cells and treated with $60 \mathrm{mg} / \mathrm{kg} \mathrm{MRX}-2843$ daily (33 days) compared with treatment with vehicle (27 days; Figure 2E), suggesting that the therapeutic effects observed in WT C57BL/6 mice are immune-mediated and dependent on adaptive immunity.

To assess the impact of MERTK inhibition in the microenvironment on leukemic burden, additional cohorts of WT and $\mathrm{Mertk}^{-/-}$mice were injected with $\mathrm{Arf}^{/-} \mathrm{p} 185^{+}$cells, and when 1 mouse in a cage exhibited 
symptoms of leukemia (typically 21-22 days after transplantation), all mice in the cage (1 mouse per treatment group) were euthanized. Following euthanasia, spleen and bone marrow were analyzed by flow cytometry to detect GFP-expressing leukemia cells. There was a significant decrease in the percentage of ALL cells in spleens and bone marrow from Mertk $^{-/}$mice (both $0.3 \%$ ) compared with WT mice $(41.3 \%-52.7 \%, P<0.0001$; Supplemental Figure 4C). Similarly, WT mice injected with $A r f^{\prime-}$ p $185^{+}$cells and treated with $60 \mathrm{mg} / \mathrm{kg} \mathrm{MRX-}$ 2843 demonstrated a significant decrease in the percentage of GFP-expressing ALL cells in bone marrow and spleens (both $0.9 \%$ ) compared with vehicle-treated mice ( $20.9 \%$ and $31.6 \%$ respectively, $P<0.01$; Supplemental Figure 4D). These data demonstrate a substantial reduction in tumor burden mediated by MERTK inhibition, presumably through immune-mediated effects on MERTK-expressing monocytes/macrophages, given that the $A r f^{\prime-}$ p $185^{+}$leukemia cells do not express MERTK. In contrast, the lower, 10-mg/kg dose of MRX-2843 had no significant impact on the fraction of GFP-expressing leukemia cells $(16.0 \%$ in the spleen and $28.9 \%$ in bone marrow; Supplemental Figure 4, C and D).

MERTK inhibition reduces expression of coinhibitory PD-L1 and PD-L2 ligands on monocytes/macrophages in the leukemia microenvironment. Previously published studies in epithelial and breast cancer cell lines demonstrated increased PD-L1 and/or PD-L2 expression in response to MERTK overexpression and decreased PD-L1 in response to shRNA-mediated MERTK inhibition $(15,16)$. To determine whether MERTK similarly impacts PD-L1 and PD-L2 on monocytes/macrophages in the leukemia microenvironment, we used flow cytometry to assess how MERTK inhibition (i.e., Mertk ${ }^{-/}$mice or MRX-2843 treatment) affects PD-L1 and PD-L2 expression on GFP-negative CD11 $\mathrm{b}^{+}$monocytes/macrophages. In both studies, the proportion of $\mathrm{CD}_{1} \mathrm{~b}^{+}$cells expressing PD-L1 and PD-L2 in the spleens (24.1\% and $12.1 \%$, respectively) and bone marrow $(4.2 \%$ and $3.2 \%$ ) of WT mice transplanted with leukemia was significantly increased relative to control mice that were not inoculated with leukemia $(2.0 \%$ in spleen in both studies, $P<0.0001$; and $0.7 \%$ or $1.1 \%$ in bone marrow, $P<0.01$; Figure 3 ), consistent with induction of an immunosuppressive microenvironment at sites of leukemic infiltration. In contrast, expression of PD-L1 and PD-L2 was not increased in the spleen $(4.8 \%)$ or marrow $(0.8 \%)$ of $\mathrm{Mertk}^{-/-}$mice inoculated with leukemia relative to uninoculated Mertk $^{-1-}$ mice (7.2\% in spleen and $1.2 \%$ in marrow; Figure 3, A and B). Similarly, in mice inoculated with leukemia and treated with a therapeutic dose of MRX-2843, the fraction of PD-L1-and PD-L2-expressing $\mathrm{CD} 11 \mathrm{~b}^{+}$cells was significantly reduced in the spleen $(1.9 \%, P<0.01)$ and marrow $(1.2 \%, P<0.05)$ relative to vehicle-treated mice (12.1\% in spleen, $3.2 \%$ in marrow; Figure $3, \mathrm{C}$ and D). Similar changes in the absolute numbers of PD-L1/PD-L2-coexpressing CD11b+ splenocytes were observed in $\mathrm{Mertk}^{-/-}$and MRX2843-treated mice; however, the absolute number of PD-L1/PD-L2-coexpressing CD11 $\mathrm{b}^{+}$bone marrow cells was not significantly increased in mice inoculated with leukemia compared with uninoculated mice (Supplemental Figure 5, A and B). Changes in the incidence of PD-L1/PD-L2-coexpressing cells largely reflected changes in PD-L2 expression. PD-L2 median fluorescence intensity (MFI) was significantly increased in spleens from WT $(1,578.0 ; P<0.0001$; Supplemental Figure 5C) and vehicle-treated (285.8; $P<0.05$; Supplemental Figure 5D) mice inoculated with leukemia compared with uninoculated control mice (245.3 and 84.7, respectively; Supplemental Figure 5, C and D). In contrast, PD-L2 MFI was not significantly different in $\mathrm{Mertk}^{-/-}$mice inoculated with leukemia (352.5) compared with uninoculated Mertk ${ }^{-/-}$ mice (410.3; Supplemental Figure 5C). Similarly, there was a significant decrease in PD-L2 MFI in spleens from mice treated with a therapeutic dose of MRX-2843 (40.58, $P<0.01$; Supplemental Figure 5D) relative to vehicle-treated control mice. Similar trends in PD-L2 MFI were observed in the bone marrow in both studies, but were not statistically significant (Supplemental Figure 5, C and D). The PD-L1 MFI was not statistically different among comparison groups (Supplemental Figure 5, E and F).

We also observed significantly increased proportions of $\mathrm{CD}_{11} \mathrm{~b}^{+}$cells in the spleens of untreated (34.5\%, $P<0.0001)$ and vehicle-treated $(28.2 \%, P<0.05)$ WT mice inoculated with leukemia relative to uninoculated control mice (11.3\% and 17.5\%; Supplemental Figure 6, A and B). In contrast, the fraction of splenic CD11b $\mathrm{b}^{+}$cells was not significantly changed in Mertk ${ }^{-1}$ mice inoculated with leukemia (10.7\%) relative to uninoculated Mertk $^{-1-}$ mice (12.5\%; Supplemental Figure 6A). Similarly, there were significantly fewer splenic CD11b cells in WT mice inoculated with leukemia and treated with $60 \mathrm{mg} / \mathrm{kg} \mathrm{MRX}-2843$ $(13.5 \%)$ compared with vehicle-treated mice $(28.2 \%, P<0.01$; Supplemental Figure $6 \mathrm{~B})$. In the bone marrow, the proportion of $\mathrm{CD}_{11 \mathrm{~b}^{+}}$cells was not significantly different in WT $(66.7 \%$ and $54.9 \%)$ or Mertk ${ }^{-1}$ mice $(47.8 \%)$ inoculated with leukemia compared with uninoculated WT (50.6\% and 46.9\%) and Mertk ${ }^{-1-}$ (48.0\%) mice, and was not significantly different in MRX-2843-treated mice (56.3\%) compared with vehicle-treated mice (54.9\%). These data suggest that MERTK inhibition reprograms the innate immune 
A

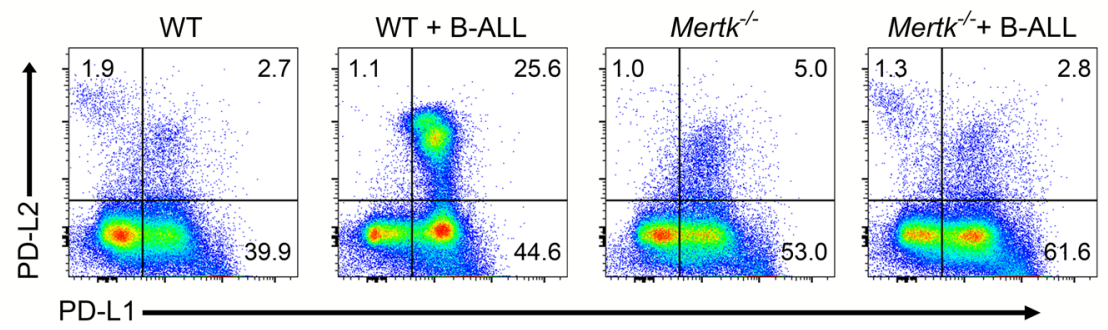

B
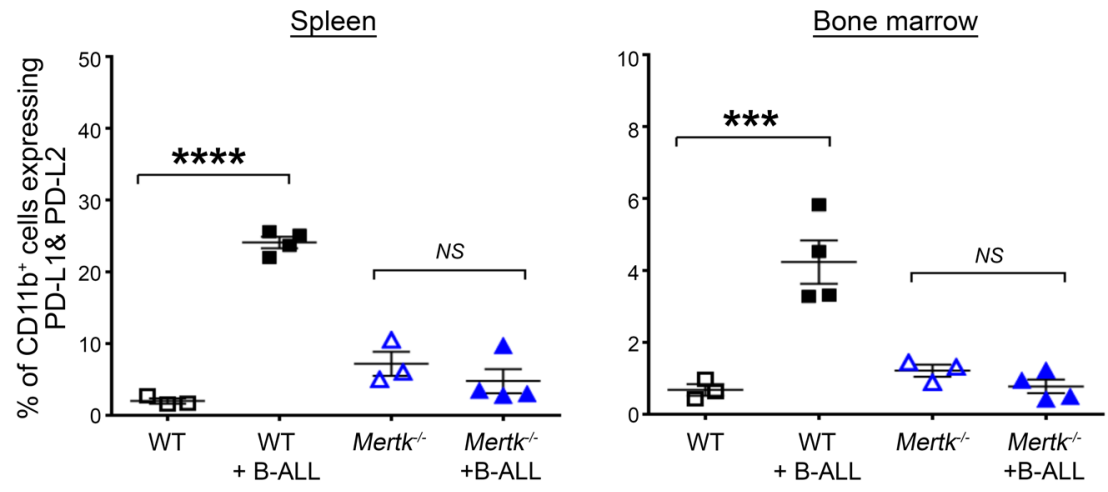

Figure 3. MERTK inhibition decreases coexpression of PD-L1 and PD-L2 on CD11b+ myeloid cells in an immunocompetent MERTK-negative B-ALL model. C57BL/6 WT or Mertk ${ }^{-1-}$ mice were injected intravenously with $\mathrm{Arf}^{-/-}$p $185^{+} \mathrm{B}-\mathrm{ALL}$ cells or saline. When symptoms of leukemia were evident in at least 1 mouse, bone marrow and spleens were harvested from those animals and their cagemates (1 per group per cage) and stained for flow cytometric analysis. (A) Representative flow cytometry profiles showing PD-L1 and PD-L2 expression on GFP- $\mathrm{CD}^{-11 \mathrm{~b}^{+}}$myeloid cells isolated from the spleens of WT and Mertk ${ }^{-1-}$ mice with and without inoculation of B-ALL. (B) Percentages of PD-L1+ PD-L2+ cells within the GFP- CD11b ${ }^{+}$population in bone marrow and spleen. (C and D) WT mice were treated with $10 \mathrm{mg} / \mathrm{kg}$ MERTK inhibitor (MRX-2843) twice daily (BID), $60 \mathrm{mg} / \mathrm{kg}$ MRX-2843 once daily, or vehicle (saline) starting 1 day after inoculation. (C) Representative flow cytometry profiles showing PD-L1 and PD-L2 expression on $\mathrm{GFP}^{-}$CD11b+ myeloid cells isolated from the spleen. (D) Percentages of PD-L1+ PD-L2+ cells within the GFP- CD11 $^{+}$population in bone marrow and spleen. Mean values and standard errors from two independent cohorts are shown. $(n=3-11$, ${ }^{*} P<0.05,{ }^{* *} P<0.01,{ }^{* * *} P<0.001,{ }^{* * * *} P<$ 0.0001 ; 1-way ANOVA).

C

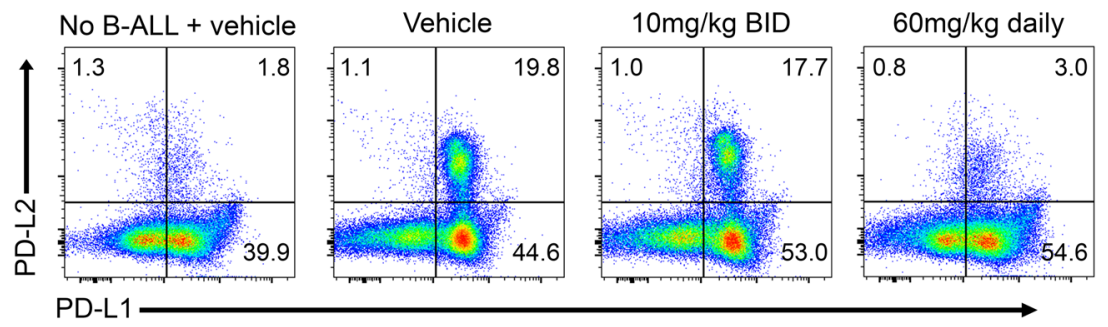

D
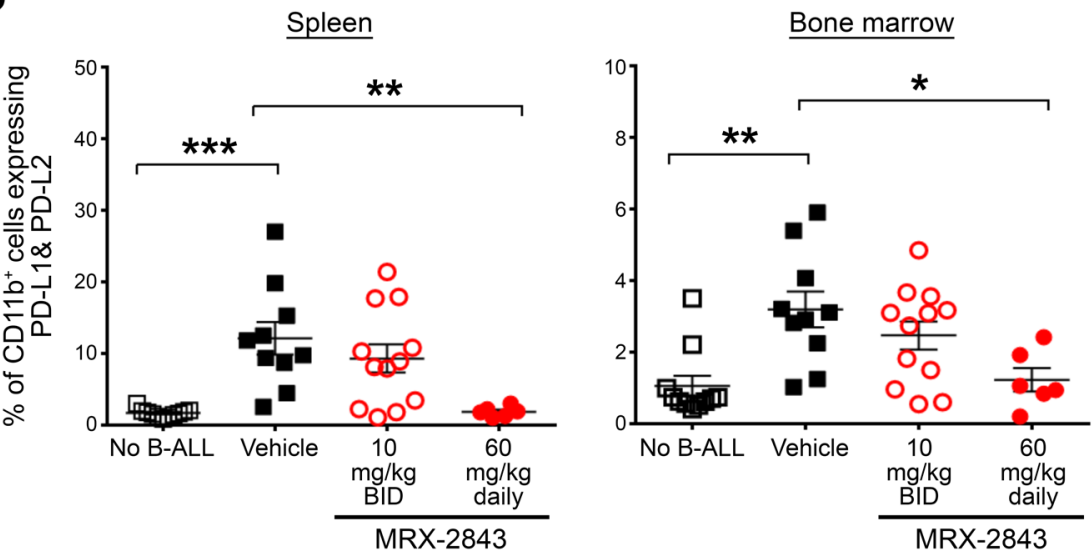

system in part by reducing immune checkpoint ligand expression on $\mathrm{CD} 11 \mathrm{~b}^{+}$monocytes/macrophages in the leukemia microenvironment.

Similar effects were observed ex vivo in mixed cultures of splenocytes and leukemia cells. The fraction of PD-L1 and PD-L2-coexpressing CD11b ${ }^{+}$B220- monocytes/macrophages was significantly increased after coculture of WT splenocytes with leukemia cells for $24(36.0 \%), 48(34.9 \%)$, and $72(36.6 \%)$ hours compared with cultures without B-ALL cells $(2.03 \%, 3.2 \%$ and $7.5 \%$; all $P<0.0001)$, or B-ALL cocultures treated with MRX-2843 (8.3\%, 15.3\%, and 9.5\%; $P<0.0001$; Figure 4 and Supplemental Figure 6C). In contrast, the 
A

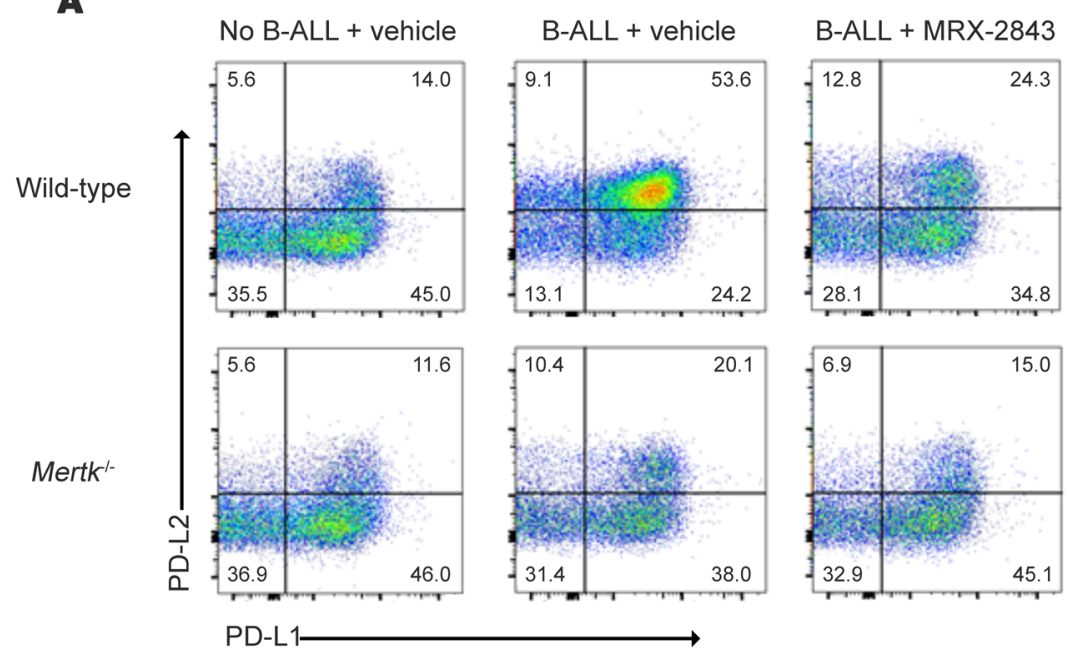

Figure 4. MERTK inhibition decreases coexpression of PD-L1 and PD-L2 on CD11b+ myeloid cells ex vivo. Splenocytes harvested from WT or Mertk ${ }^{-/-}$mice were cultured at a 1:1 ratio with $\mathrm{Arf}^{-1-}$ p185 $\mathrm{B}-\mathrm{ALL}$ cells and treated with $200 \mathrm{nM}$ MRX-2843 or vehicle (DMSO) for 72 hours. The indicated cell surface proteins were quantitated by flow cytometry. (A) Representative dot plots showing PD-L1+ PD-L2+ cells within the GFP-CD11b+ population. (B) Graphical representation of the percentage of GFP-CD11 b+ cells coexpressing PD-L1+ and PD-L2+ Mean values and standard errors from 2 independent cohorts are shown. ( ${ }^{* * *} P<0.0001 ; 2$-way ANOVA).

\section{B}

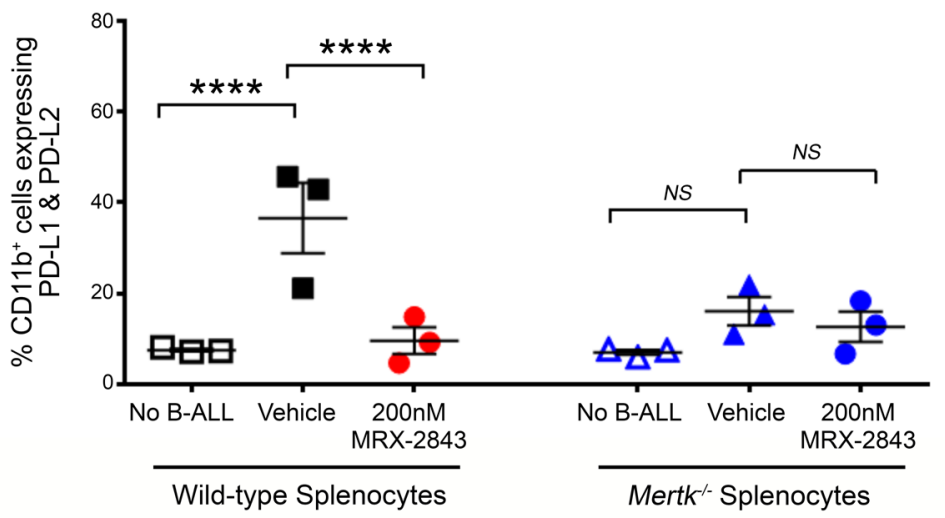

incidence of PD-L1/PD-L2-coexpressing CD11 $\mathrm{b}^{+}$monocyte/macrophages was not significantly different in cultures of splenocytes from Mertk ${ }^{--}$mice in the presence (16.0\%) or absence (7.0\%) of leukemia cells, or when treated with MRX-2843 (12.6\%; Figure 4B). Thus, when MERTK was absent, MRX-2843 had no effect, consistent with MERTK inhibition as a mechanism of MRX-2843 immunomodulatory activity.

MERTK inhibition reduces expression of coinhibitory PD-1 receptor on T cells in the leukemia microenvironment. Given the decreased PD-L1 and PD-L2 expression on monocytes/macrophages in the leukemia microenvironment in response to MERTK inhibition, we assessed the impact of MERTK inhibition (Mertk $\mathrm{K}^{-1-}$ and MRX-2843 treatment) on expression of the PD- 1 coinhibitory receptor on $\mathrm{CD}^{+}$and $\mathrm{CD} 8^{+} \mathrm{T}$ cells in the spleen and bone marrow using flow cytometry. In WT mice inoculated with leukemia, the fractions of CD4 ${ }^{+}$ and $\mathrm{CD} 88^{+} \mathrm{T}$ cells expressing PD-1 in spleens ( $74.2 \%$ and $49.2 \%$, respectively) and bone marrow $(65.9 \%$ and $73.0 \%)$ were significantly increased compared with those in spleens $(10.0 \%$ and $4.1 \%)$ and bone marrow (36.4\% and $8.5 \%)$ from uninoculated control mice $(P<0.0001$; Figure $5, \mathrm{~A}$ and B, and Supplemental Figure 7A). However, the fraction of $\mathrm{PD}-1-$ expressing $\mathrm{CD} 4^{+}$and $\mathrm{CD}^{+} \mathrm{T}$ cells was not significantly different in spleens (28.3\% and 7.2\%) and bone marrow (66.2\% and 29.2\%) from $\mathrm{Mertk}^{-1-}$ mice inoculated with leukemia compared with spleens (20.0\% and 4.7\%) and bone marrow (62.2\% and $12.4 \%$ ) from uninoculated Mertk $^{-1}$ mice. Similarly, PD-1-expressing $\mathrm{CD}^{+}$and $\mathrm{CD} 8^{+} \mathrm{T}$ cells were significantly reduced in spleens $(14.7 \%$ and $7.9 \%$, respectively) and bone marrow $(45.3 \%$ and $12.0 \%)$ from mice inoculated with leukemia and treated with $60 \mathrm{mg} / \mathrm{kg}$ MRX-2843 daily relative to spleens $(53.4 \%$ and $45.5 \% ; P<0.001)$ and bone marrow $(58.6 \%, P$ $<0.05$ and 55.8\%, $P<0.001$ ) from vehicle-treated controls (Figure 5, C and D, and Supplemental Figure 7B). Alterations in PD-1 expression correlated with an increase in the median intensity of PD-1 expression on T cells (Supplemental Figure 7, C and D), without a significant increase in the total number of PD-1-expressing $\mathrm{T}$ cells in tissues with leukemia infiltration (Supplemental Figure 8). The concurrent increases in PD-L1 and 
A
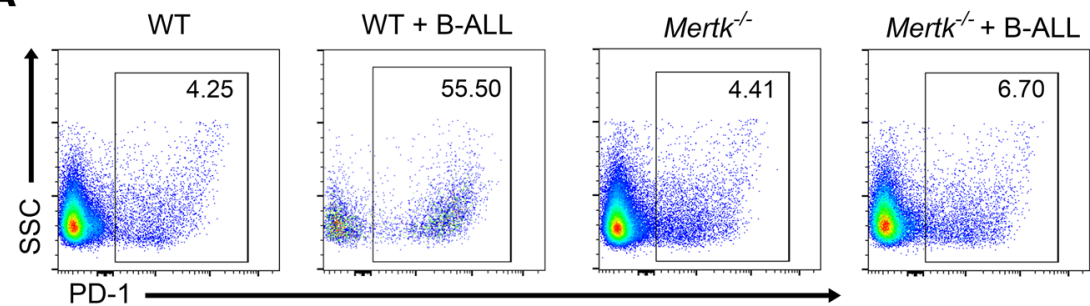

B

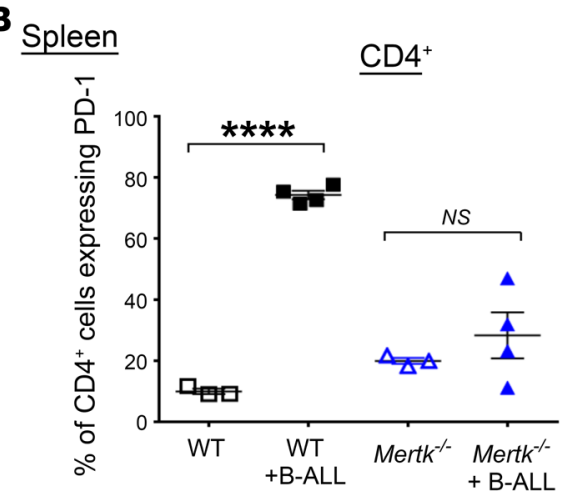

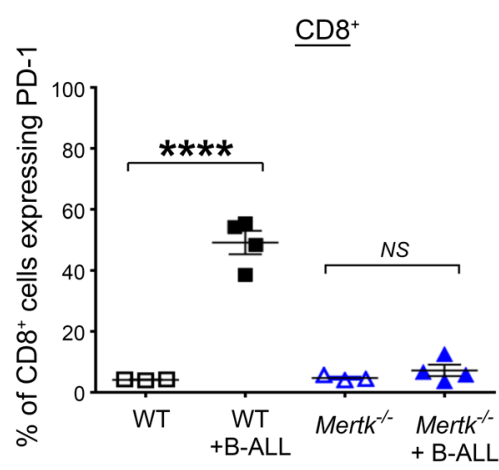

Figure 5. MERTK inhibition decreases expression of PD-1 on CD8 ${ }^{+}$cells in a syngeneic MERTK-negative B-ALL model. C57BL/6 WT or Mertk ${ }^{-1-}$ mice were injected intravenously with $\mathrm{Arf}^{-1-}$ p $185^{+} \mathrm{B}-\mathrm{ALL}$ cells or saline, and bone marrow and spleens were harvested and stained for flow cytometric analysis as described in Figure 3. (A) Representative flow cytometry profiles showing $\mathrm{PD}-1$ expression on $\mathrm{CD} 8^{+} \mathrm{T}$ cells within the GFP-CD3+ population in spleens of WT and Mertk $^{-1-}$ mice. (B) Percentages of PD-1+ cells within the $\mathrm{GFP}^{-} \mathrm{CD3}^{+} \mathrm{CD}^{+}$and $\mathrm{CD} 8^{+}$populations in spleens of WT and Mertk $^{-1-}$ mice. (C) Representative flow cytometry profiles showing PD-1 expression on CD8 ${ }^{+}$ $\mathrm{T}$ cells within the GFP-CD3+ population isolated from the spleens of WT mice treated with MRX-2843 or vehicle (saline). (D) Percentages of PD-1+ cells within the $\mathrm{GFP}^{-} \mathrm{CD}^{+} \mathrm{CD}^{+}$and $\mathrm{CD} 8^{+}$population in spleens of WT mice treated with MRX-2843. Mean values and standard errors from 2 independent cohorts are shown. $(n=3-12) .{ }^{* *} P<0.001,{ }^{* * *} P<0.0001$; 1-way ANOVA)

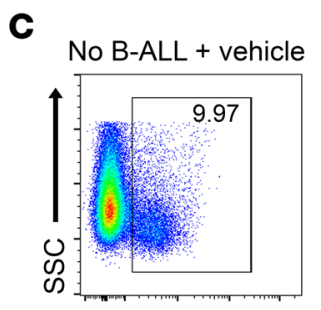

PD-1

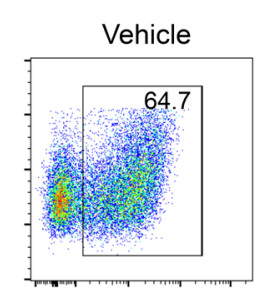

$\longrightarrow$
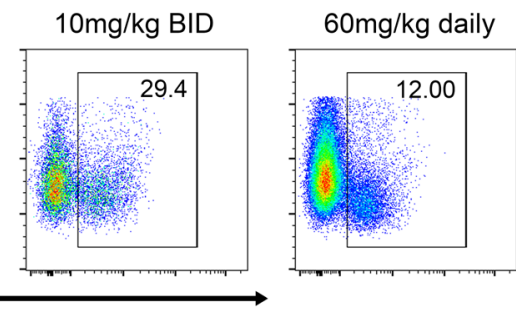

D

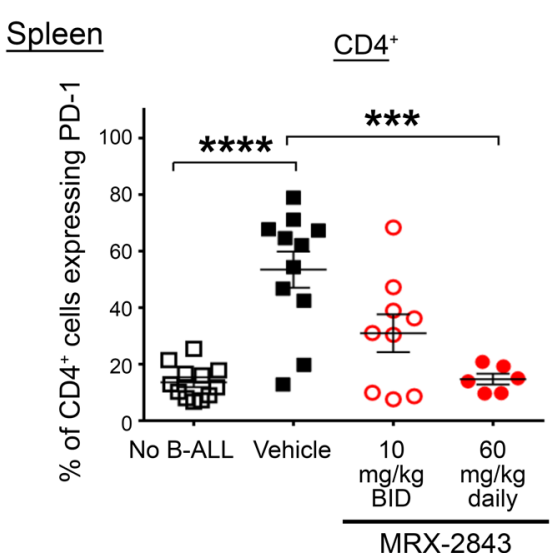

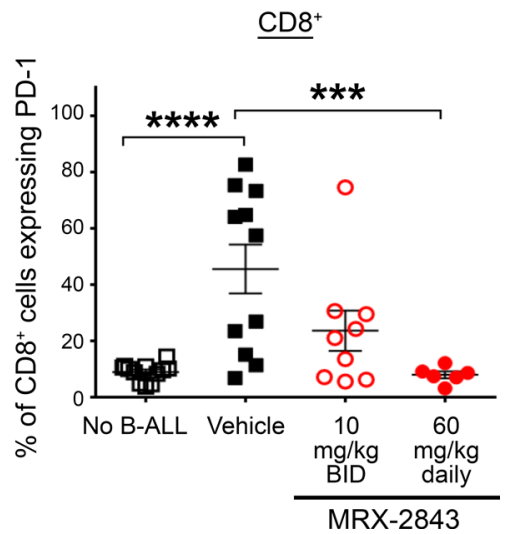

PD-L2 expression on CD11 $\mathrm{b}^{+}$monocytes/macrophages and PD-1 expression on T cells that we observed in leukemic mice are consistent with induction of an immunosuppressed state in the leukemia microenvironment, and these effects were abrogated in response to MERTK genetic deletion or pharmacologic inhibition.

MERTK inhibition increases $T$ cell activation in the leukemia microenvironment. To determine whether changes in PD-1 expression were accompanied by functional changes in T cell activation, T cells from WT mice were stimulated with plate-bound anti-CD3, then added to splenocyte and leukemia cocultures for an additional 24 hours, and intracellular IFN- $\gamma$ and TNF- $\alpha$ levels were assessed as markers of T cell activation using flow cytometry. The fraction of activated (IFN- $\gamma^{+}$and TNF- $\left.\alpha^{+}\right) \mathrm{CD} 4^{+}$and $\mathrm{CD} 8^{+} \mathrm{T}$ cells significantly increased in cocultures treated with MRX-2843 (44.2\% and 51.1\%, respectively) compared with $\mathrm{CD}^{+}$and $\mathrm{CD} 8^{+} \mathrm{T}$ cells in cocultures treated with vehicle $(27.4 \%$ and $36.8 \%, P<0.0001$; Figure 6 , A and C, upper panels, and 
A

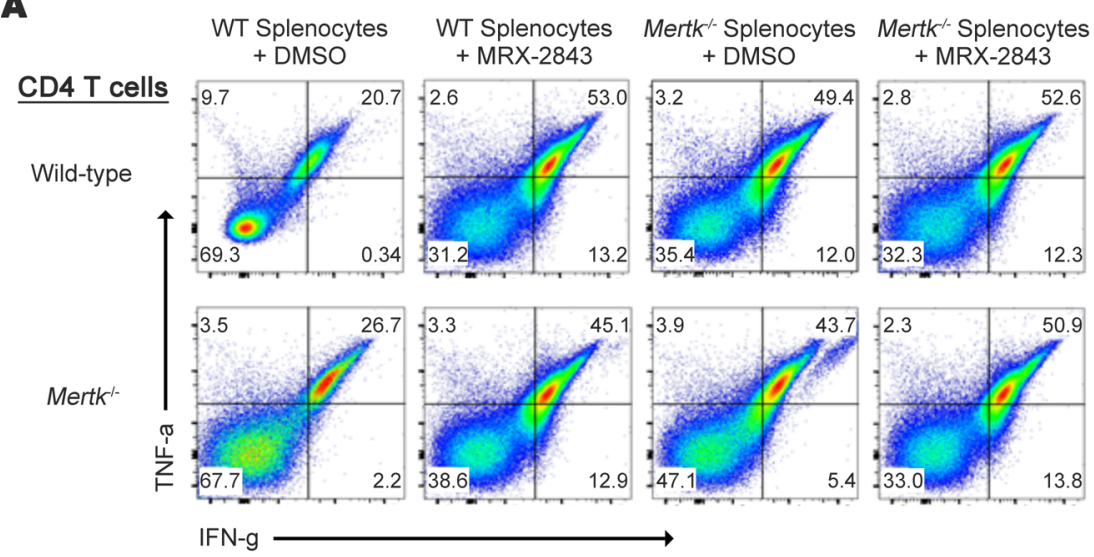

C

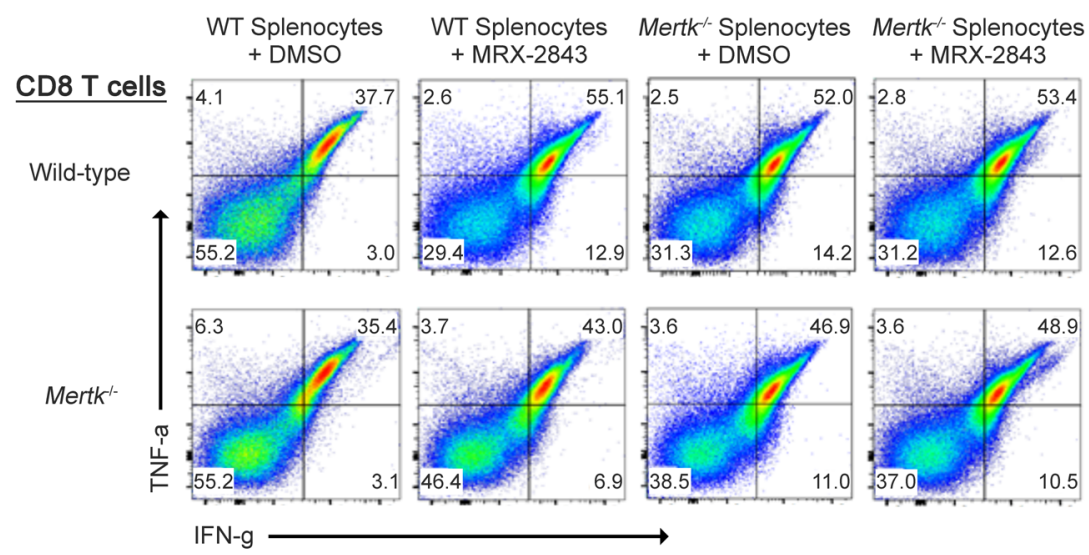

B

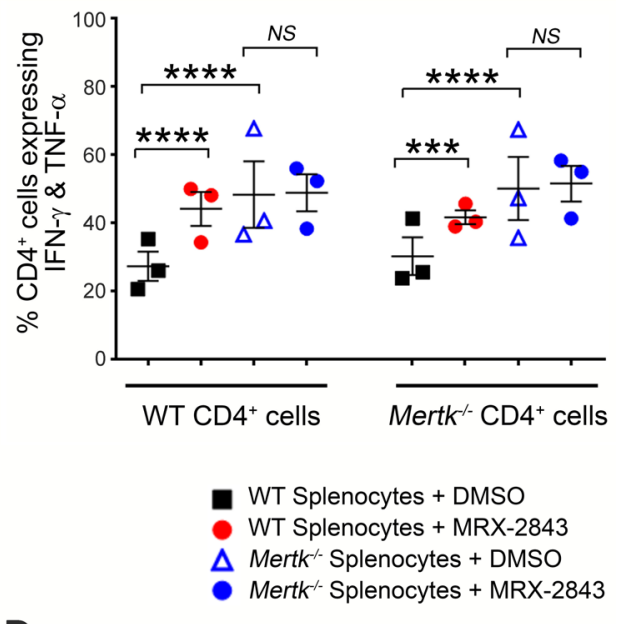

D

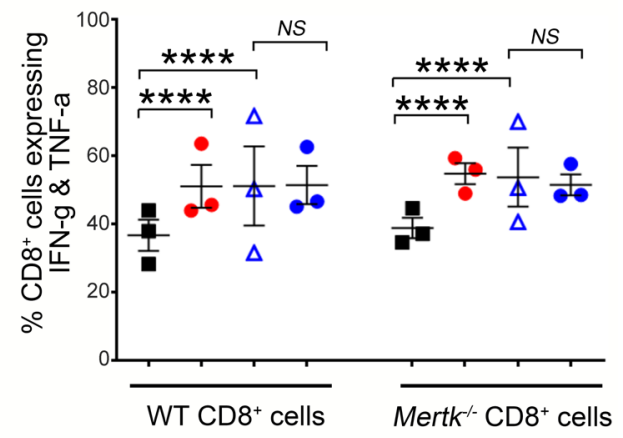

Figure 6. MERTK inhibition indirectly increases $\mathrm{CD4}^{+}$and $\mathrm{CD8}{ }^{+} \mathbf{T}$ cell activation ex vivo. $\mathrm{CD4} 4^{+}$and $\mathrm{CD} 8^{+} \mathrm{T}$ cells were isolated from spleens from $\mathrm{C57BL/6}$ WT or Mertk ${ }^{-1-}$ mice and stimulated for 24 hours with anti-CD3 antibody, then cultured at a 2:1:1 ratio with Arf ${ }^{-1-}$ p185 $5^{+}$-ALL cells and WT or Mertk ${ }^{-/-}$ splenocytes and treated with 200 nM MRX-2843 or vehicle (DMSO) for 72 hours. Production of IFN- $\gamma$ and TNF- $\alpha$ in CD4 ${ }^{+}$and CD8 $8^{+}$T cells was measured by flow cytometry. (A and C) Representative dot plots showing IFN- $\gamma$ and TNF- $\alpha$ levels in CD4+ (A) and CD8+ (C) T cells. (B and D) Graphical representation of the percentage of CD4+ and CD8 ${ }^{+}$T cells expressing both IFN- $\gamma$ and TNF- $\alpha$. Mean values and standard errors from 3 independent experiments are shown. ${ }^{* *} P<0.001,{ }^{* * *} P<0.0001 ; 2$-way ANOVA.

Figure 6, B and D, left panels). Similarly, activation of $\mathrm{CD} 4^{+}$and $\mathrm{CD} 8^{+} \mathrm{T}$ cells was increased in cocultures with Mertk $^{--}$splenocytes (48.3\% and $51.2 \%$, respectively) compared with coculture with WT splenocytes $(P<0.0001)$, and activation was not further augmented by treatment with MRX-2843 $(48.9 \%$ and $51.5 \%)$. Similar results were obtained when $\mathrm{T}$ cells isolated from $\mathrm{Mertk}^{-1-}$ mice were used for these assays (Figure 6, A and C, lower panels and Figure 6, B and D, right panels). Thus, the effect of MERTK inhibition on T cell activation was dependent on the presence of MERTK on antigen-presenting splenocytes, but not $\mathrm{T}$ cells.

MERTK inhibition decreases splenic FOXP3-expressing Tregs. We next assessed the incidence of CD4+ FOXP $3^{+}$Tregs in mice with and without MERTK inhibition (Mertk ${ }^{--}$and MRX-2843 treatment) using flow cytometry. In both studies, there was a significant increase in the percentage of splenic Tregs in WT mice inoculated with leukemia $(36.0 \%$ and $18.3 \%)$ relative to uninoculated control mice $(6.3 \%$ and $4.8 \% ; P<0.001 ;$ Figure 7). In contrast, proportions of splenic Tregs were not significantly altered in Mertk $^{-1-}$ mice inoculated with leukemia (11.3\%) relative to uninoculated control mice $(9.3 \%$; Figure 7 , $\mathrm{A}$ and $\mathrm{B})$. Similarly, there was a significant decrease in the percentage of splenic $\mathrm{CD} 4^{+} \mathrm{FOXP} 3^{+}$Tregs in mice treated with a therapeutic dose of MRX-2843 $(5.1 \% ; P<0.01)$ compared with vehicle-treated mice (Figure 7, $\mathrm{C}$ and D). The percentage of splenic Tregs was also significantly decreased in response to treatment with the subtherapeutic dose of MRX-2843 $(9.0 \% ; P<0.05)$. These changes in Treg populations in the spleen were accompanied by similar changes in the proportion of $\mathrm{CD}^{+}$lymphocytes, but were independent of significant changes in the absolute number of $\mathrm{CD}^{+}$lymphocytes or $\mathrm{CD} 4^{+} \mathrm{FOXP} 3^{+}$ Tregs (Supplemental Figure 9). Additionally, these changes were independent of tumor burden, given 
A
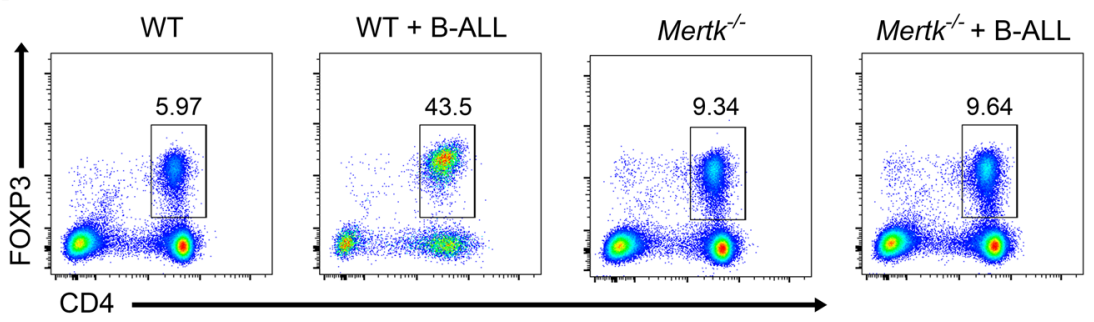

B

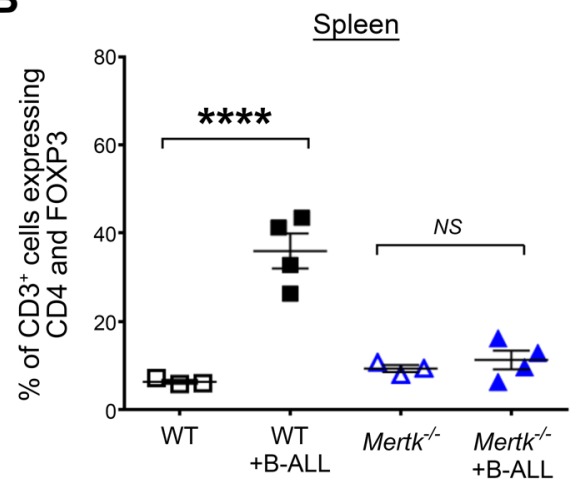

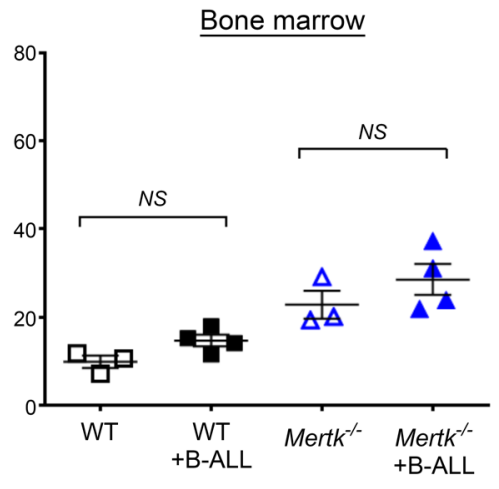

Figure 7. MERTK inhibition decreases splenic Treg populations in a MERTK-negative B-ALL allograft model. C57BL/6 WT or Mertk ${ }^{-1-}$ mice were injected intravenously with $\mathrm{Arf}^{-/-}$p $185^{+} \mathrm{B}-\mathrm{ALL}$ cells or saline, and bone marrow and spleens were harvested and stained for flow cytometric analysis as described in Figure 3. (A) Representative flow cytometry profiles showing $\mathrm{CD}^{+} \mathrm{FOXP3}^{+}$Tregs within the $\mathrm{GFP}^{-} \mathrm{CD3}^{+}$population isolated from the spleens of WT and Mertk ${ }^{-1-}$ mice. (B) Percentages of $\mathrm{CD}^{+}{ }^{+} \mathrm{FOXP3}^{+}$cells within the $\mathrm{GFP}^{-} \mathrm{CD}^{+}$ population in bone marrow and spleens of WT and Mertk $^{-1-}$ mice. (C) Representative flow cytometry profiles identifying the $\mathrm{CD} 4^{+} \mathrm{FOXP3^{+ }} T$ regulatory cells within the $\mathrm{GFP}^{-} \mathrm{CD3}^{+}$population isolated from the spleens of WT mice treated with MERTK inhibitor MRX-2843 or vehicle (saline). (D) Percentages of $\mathrm{CD}^{+} \mathrm{FOXP3}^{+}$cells within the GFP-CD3 ${ }^{+}$ population in bone marrow and spleens of WT mice treated with MRX-2843. Mean values and standard errors from 2 independent cohorts are shown. ( $n=$ $3-12,{ }^{*} P<0.05$, ${ }^{* *} P<0.01,{ }^{* *} P<0.001,{ }^{* * * *} P<$ $0.0001 ; 1$-way ANOVA).

\section{C}

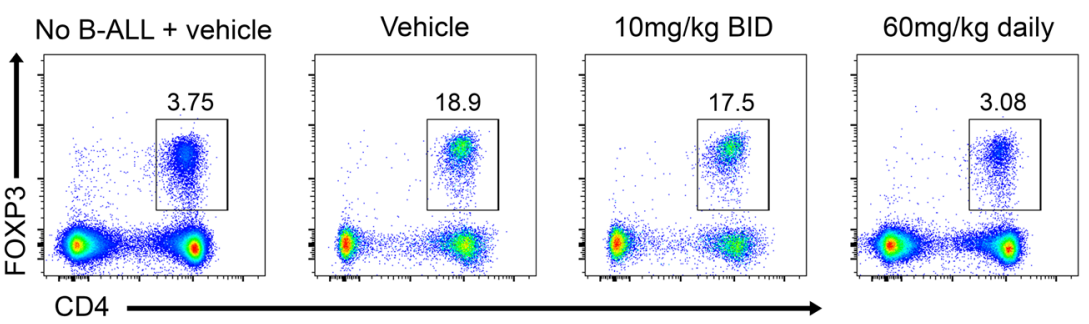

D
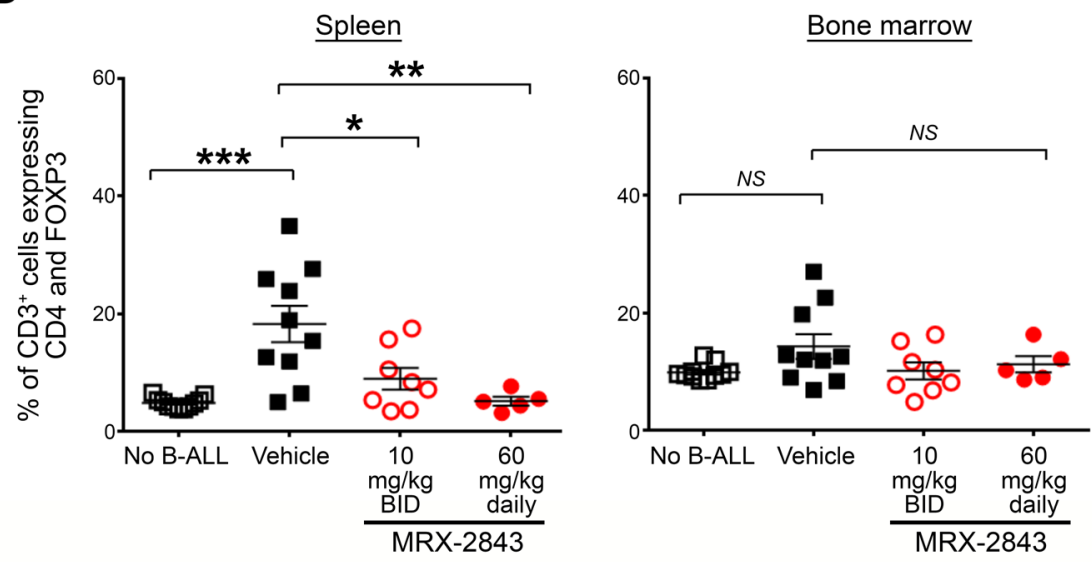

the significant decrease in Tregs in mice treated with the subtherapeutic $10 \mathrm{mg} / \mathrm{kg}$ dose of MRX-2843 $(P<0.05$, Figure 7D). In contrast to the significant changes in the fraction of Tregs in the spleen in response to MERTK inhibition, the percentage of $\mathrm{CD} 4^{+} \mathrm{FOXP}^{+}$cells in the bone marrow was not significantly altered in response to the presence of leukemic blasts and was not consistently changed in response to MERTK inhibition. There was no significant difference in the incidence of Tregs in bone marrow from $\mathrm{Mertk}^{-/-}$mice with (28.6\%) or without leukemia (22.8\%), or in mice treated with MRX$2843(11.2 \%)$ relative to vehicle-treated mice (14.3\%; Figure 7, B and D). 


\section{Discussion}

We previously validated MERTK as a potential therapeutic target in ALL using both RNA interference and MERTK inhibitors $(1-3,30)$. Here we demonstrate therapeutic effects in immunocompromised and immunocompetent ALL models mediated by MERTK inhibition using a small molecule, MRX2843 , that has recently advanced to clinical application. MRX-2843 administered at a dose of $75 \mathrm{mg} / \mathrm{kg}$ mediated sustained inhibition of MERTK phosphorylation in bone marrow leukemia cells in vivo over 24 hours. With continuous pharmacodynamic inhibition, disease burden was significantly reduced and survival was prolonged by 2- to 3 -fold in an immunocompromised murine xenograft model of MERTK-expressing human leukemia. These data demonstrate direct anti-leukemia activity in response to treatment with MRX-2843 and are consistent with our previous studies using earlier MERTK inhibitors and shRNA-mediated MERTK inhibition. Given that neither Mertk-knockout mice nor mice treated with a therapeutic dose of MRX-2843 exhibit profound anemia, thrombocytopenia, or neutropenia (24), it may be possible to target MERTK in leukemia to improve therapeutic efficacy (1) and potentially lower the dose of cytotoxic chemotherapy required for cure.

In macrophages, MERTK expression has been correlated with anti-inflammatory signaling (13, 14, 27) and a wound-healing M2 phenotype (9). MERTK activation in innate immune cells in response to phosphatidylserine binding attenuates Toll-like receptor signaling through several mechanisms, including

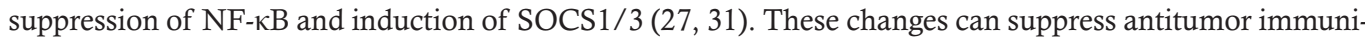
ty. Indeed, genetic deletion of MERTK in mice transplanted with syngeneic breast cancer, colon cancer, or melanoma slowed tumor growth, inhibited metastases, and altered the immune microenvironment, including enhanced expression of proinflammatory IL-12 and IL-6 in tumor-infiltrating monocytes/macrophages $(13,14)$. Given these findings, we hypothesized that MERTK inhibition would reverse the immunosuppressive leukemia microenvironment to promote an immune-mediated therapeutic response.

In WT mice, treatment with MRX-2843 did not significantly alter proportions of circulating monocytes, granulocytes, and lymphocytes, despite a moderate decrease in MEPs. Thus, the capacity for an immune response was demonstrably intact. Indeed, treatment with MRX-2843 reduced leukemic burden and prolonged survival in immunocompetent mice bearing a MERTK-negative B-ALL, with $20 \%$ of mice exhibiting long-term survival and no overt evidence of disease. To determine whether increased survival in this model is due to inhibition of host MERTK, we inoculated the same MERTK-negative ALL cells into Mertk $^{-/-}$mice and monitored survival. In the context of a Mertk ${ }^{-/-}$microenvironment, there was an even more profound effect on leukemogenesis, with all but one of the $\mathrm{Mertk}^{-/-}$mice surviving for 150 days after transplant, with no overt evidence of disease, compared with a 21-day median survival for WT mice. In contrast, treatment with MRX-2843 had no significant impact on leukemia progression in immunocompromised NSG mice. These data suggest an immune-mediated mechanism for antitumor activity in this model and demonstrate the potential utility of therapies targeting MERTK for immuno-oncology applications in the context of ALL, irrespective of whether MERTK is expressed on the leukemic blasts themselves.

Interestingly, there was a significant increase in the fraction of $\mathrm{CD}_{11} \mathrm{~b}^{+}$monocytes/macrophages coexpressing PD-L1 and PD-L2 at sites of leukemia infiltration, and inhibition of MERTK through genetic deletion or using therapeutic doses of a MERTK inhibitor abrogated this response. In the spleen, changes in the proportion of $\mathrm{PD}-\mathrm{L} 1 / \mathrm{PD}-\mathrm{L} 2-$ coexpressing $\mathrm{CD} 11 \mathrm{~b}^{+}$cells primarily reflected changes in PD-L2 expression, while in the bone marrow changes in both ligands contributed. Similar changes in the incidence of PD-L1/PD-L2-expressing monocytes/macrophages were observed when WT and Mertk-/- splenocytes were cocultured ex vivo with leukemia cells and/or MRX-2843. The precise mechanism by which MERTK inhibition decreases PD-L1 and PD-L2 is unclear; however, others have demonstrated that induction of PD-L1 and PD-L2 in response to MERTK overexpression in 293FT cells and shRNA-mediated MERTK inhibition reduced PD-L1 in breast cancer cells $(15,16)$. In these studies, PD-L1 was also augmented in response to apoptotic cell ingestion, suggesting upregulation of coinhibitory ligands in response to MERTK activation during efferocytosis, the homeostatic process that prevents autoimmunity. MERTK also promotes the alternatively activated M2 macrophage phenotype (9), and previous studies demonstrated upregulation of PD-L2 expression on IL-4-induced M2 macrophages (32), suggesting a potential link between MERTK and PD-L2 expression in this context. Though the functional significance of PD-L1 and PD-L2 coexpression on leukemia-associated macrophages has not been specifically studied, there is a significant correlation between PD-L1 and PD-L2 expression in a variety of tumor types, and the 2 ligands frequently colocalize in tumor-associated macrophages (33). Additionally, PD-L1 and PD-L2 are both independent prognostic 
markers in several tumor types $(34,35)$, and PD-L1/PD-L2 coexpression has been associated with significantly shorter overall survival in patients with esophageal cancer compared with patients whose tumors did not express PD-1 ligands (21).

Although MERTK is not normally expressed in murine T cells $(36,37)$, MERTK inhibition decreased $\mathrm{PD}-1$ expression on $\mathrm{CD}^{+}$and $\mathrm{CD} 8^{+} \mathrm{T}$ cells in the leukemia microenvironment and decreased splenic Treg populations. Treg populations were also decreased in response to treatment with the subtherapeutic 10-mg/ $\mathrm{kg}$ dose of MRX-2843, indicating that partial reduction of these cells alone is not sufficient for anti-leukemia immunity in response to MERTK inhibition. In ex vivo coculture assays, $\mathrm{CD} 4^{+}$and $\mathrm{CD} 8^{+} \mathrm{T}$ cells exhibited increased production of the intracellular T cell activation cytokines IFN- $\gamma$ and TNF- $\alpha$ in response to MERTK inhibition, providing what we believe to be the first evidence of changes in $\mathrm{T}$ cell function downstream of MERTK inhibition. Increased T cell activation was observed when MERTK was inhibited on splenocytes, but not when it was inhibited on T cells. These data support a model whereby MERTK functions in monocytes/macrophages to promote expression of the coinhibitory ligands PD-L1 and PD-L2 and indirectly inhibits $\mathrm{T}$ cell activity through a paracrine mechanism leading to increased expression of PD-1 on $\mathrm{CD}^{+}$and $\mathrm{CD} 8^{+} \mathrm{T}$ cells, decreased $\mathrm{T}$ cell activation, and a tumor-permissive environment. Taken together, these findings demonstrate immunosuppressive roles for MERTK in the leukemia microenvironment (Figure 8A). Additionally, induction of MERTK expression has been described in human $\mathrm{T}$ cells following persistent $\mathrm{T}$ cell receptor stimulation, suggesting that in some cases MERTK inhibition might directly impact $\mathrm{T}$ cell function (10). Regardless, it is clear that MERTK inhibition reverses the immunosuppressive milieu by decreasing the proportion of PD-L1 and PD-L2-coexpressing monocytes/macrophages and altering the microenvironment in a manner leading to a decreased incidence of Tregs and reduced expression of PD-1 on $\mathrm{CD}^{+}$and $\mathrm{CD}^{+} \mathrm{T}$ cells (Figure 8B). Many studies investigating therapies targeting coinhibitory receptors have focused on PD-L1 and PD-L2 expression on malignant cells. Our data indicate that evaluation of coinhibitory ligand expression in the leukemic innate immune microenvironment is also warranted. Further evaluation of the immune-mediated effects of MERTK inhibition is needed, including analysis of the downstream signaling and mechanisms that alter coinhibitory ligand/receptor expression.

MRX-2843 is a dual MERTK and FLT3 kinase inhibitor. While we cannot eliminate the possibility that some of the antitumor activity mediated by MRX-2843 in animal models is due to FLT3 or off-target kinase inhibition, there are several lines of evidence that argue against this. The direct anti-leukemia activity against 697 B-ALL xenografts recapitulates the effects of shRNA-mediated MERTK knockdown in the same model (26). In addition, although 697 cells express low levels of FLT3, they do not carry the activating FLT3 mutations that have been associated with leukemogenesis (38). Similarly, the immunomodulatory and therapeutic effects of MRX-2843 in immunocompetent mice transplanted with the $\mathrm{Arf}^{\prime-}$ p185 murine B-ALL cell line were recapitulated in Mertk ${ }^{-/}$mice, and treatment with MRX-2843 had no therapeutic effect against the murine B-ALL in immunocompromised mice. Together these data and observations implicate MERTK inhibition as the mechanism of both direct and immune-mediated MRX-2843 therapeutic activity.

In conclusion, the data presented demonstrate a dual therapeutic effect of MERTK inhibition mediated by both direct anti-leukemia activity on MERTK-expressing ALL cells and induction of antitumor immunity in the leukemia microenvironment. This dual mechanism of action implicates MERTK inhibition as a particularly attractive strategy for treatment of MERTK-expressing tumors. Additionally, these are the first reported studies to our knowledge demonstrating prolonged survival mediated by pharmacologic inhibition of MERTK in the leukemia microenvironment in an immunocompetent model, suggesting both direct and indirect roles for MERTK in immune suppression. Thus, assessment of the impact of MERTK inhibition in the cancer microenvironment should extend beyond the solid tumor models that have previously been reported to include the niches that nurture leukemia cells and prevent cure of hematologic malignancies.

\section{Methods}

Cell culture. The 697 cell line was obtained from the German Collection of Microogranisms and Cell Cultures. The J774 cell line was obtained from the ATCC. Arf ${ }^{-}$BCR-ABL $185^{+}$ALL cells expressing GFP were generated by Richard Williams (29). The luciferase-expressing 697 cell line $\left(697^{\text {luct }}\right)$ was generated as previously described (39). All leukemia cell lines were cultured in RPMI medium supplemented with $10 \%$ FBS, penicillin/streptomycin, and L-glutamine. The J774 macrophage cell line was cultured in DMEM 
A
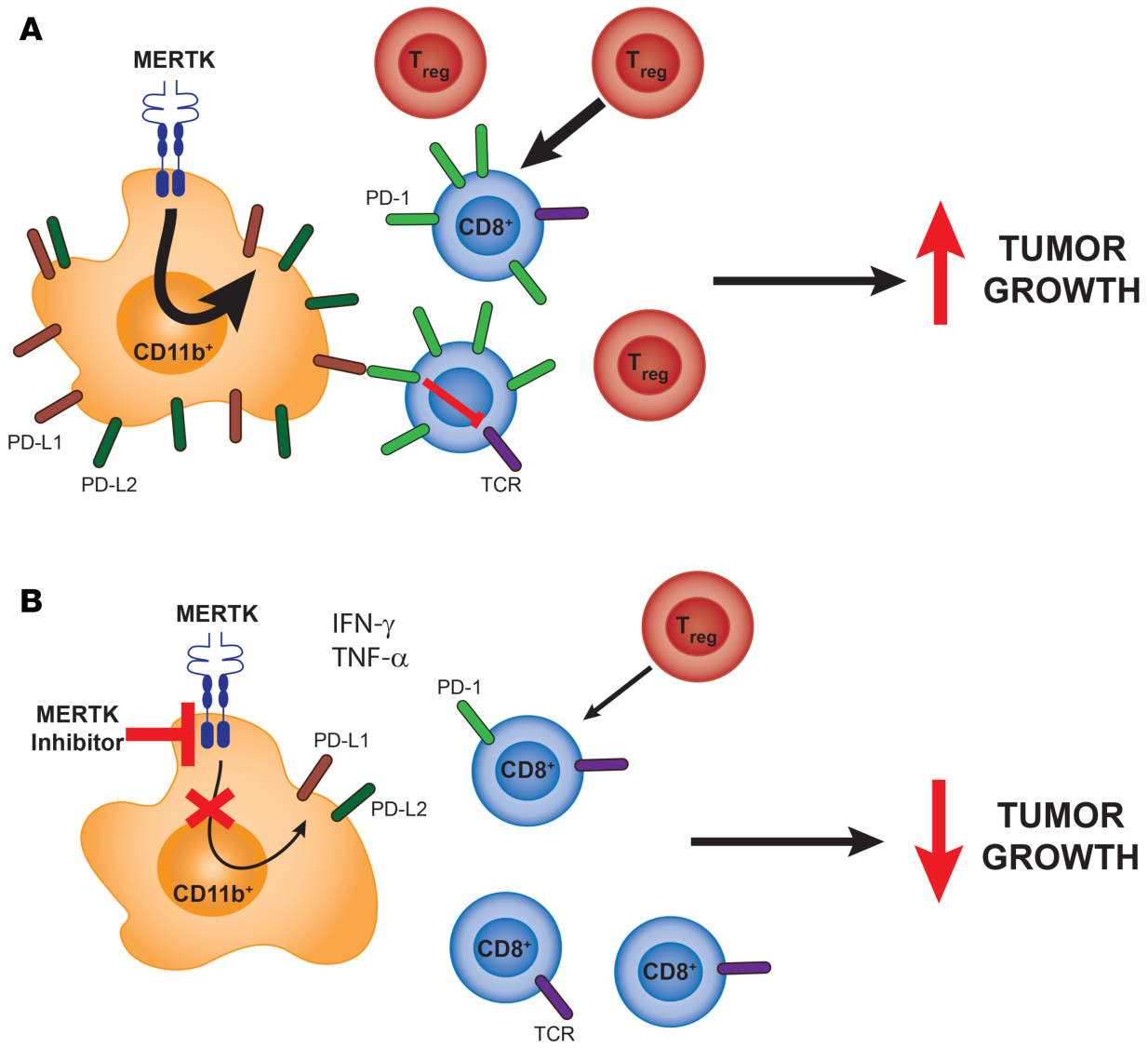

Figure 8. MERTK-dependent regulation of innate immunity decreases coinhibitory ligand/receptor expression and Tregs in the leukemia microenvironment, leading to reduced leukemic burden. Model depicting roles for MERTK in innate immune responses in the leukemia microenvironment. (A) In the presence of ALL, MERTK induces coexpression of the coinhibitory ligands PD-L1 and PD-L2 on CD11b+ monocytes/macrophages, and indirectly induces expression of the coinhibitory receptor PD-1 on $\mathrm{CD}^{+} \mathrm{T}$ cells and promotes an increased incidence of Tregs. (B) In response to MERTK inhibition, coexpression of PD-L1 and PD-L2 decreases on CD11 b+ cells, expression of PD-1 on MERTK-negative T cells decreases, and regulatory $T$ cells are reduced, leading to $T$ cell activation and induction of anti-leukemia immunity and ultimately resulting in reduced leukemic burden. TCR, T cell receptor.

medium supplemented with 10\% FBS and penicillin/streptomycin. Human leukemia cell line identities were confirmed using short tandem repeat microsatellite locus analysis.

Pharmacodynamic studies. Six- to 8-week-old NOD.Cg-Prkdc $c^{\text {scid }} I l 2 \mathrm{rg}^{\text {tm } I W j l} / \mathrm{SzJ}$ (NSG) mice were purchased from the Jackson Laboratory or bred in-house and maintained under sterile conditions. Mice were injected intravenously with $2 \times 10^{6} 697$ cells. Fourteen days after xenograft transplantation, mice were treated with a single $10-\mathrm{ml} / \mathrm{kg}$ oral dose of vehicle (saline) or the MERTK inhibitor MRX-2843 $(24,25)$. Bone marrow was flushed from femurs with RPMI medium containing $20 \% \mathrm{FBS}$ and $240 \mu \mathrm{M}$ pervanadate, then incubated at room temperature for 10 minutes prior to preparation of cell lysates for detection of phosphorylated and total MERTK proteins (25). Densitometry was performed using NIH ImageJ software.

B-cell ALL xenograft model and bioluminescence imaging. Luciferase-expressing 697 cells (40) were injected via tail vein into NSG mice to establish xenografts. MRX-2843 (24) or vehicle was administered by oral gavage in a volume of $10 \mathrm{ml} / \mathrm{kg}$. Leukemia burden was monitored twice weekly using a Xenogen IVIS200 imaging system (40). Bioluminescence was measured in units of photons/second using Living Image software (PerkinElmer).

Immunoblot analysis. Cell lysates were prepared and samples were resolved as previously described (41). For analysis of phosphorylated MERTK, MERTK protein was immunoprecipitated from cell lysates and phosphorylated, and total proteins were detected by immunoblot as previously described (25). Immunoblots were probed with the following primary antibodies: anti-human actin (sc-1616, Santa Cruz Biotechnology Inc.); anti-human MERTK [Y323] (ab52968, Abcam); anti-mouse tubulin (2125S, Cell Signaling Technology); anti-mouse MERTK (AF591, R\&D Systems), anti-FLT3/FLK2 (3462S, Cell Signaling Technology).

Murine B cell ALL model. Mertk-null (B6;129-Mertk ${ }^{\text {tmlGrl } / J) ~ m i c e ~(27) ~ w e r e ~ b a c k c r o s s e d ~ w i t h ~ C 57 B L / 6 J ~}$ mice for more than 12 generations (Mertk ${ }^{-/}$), and Mertk genotype was verified prior to use. WT C57BL/6J and NSG mice were purchased from the Jackson Laboratory and bred in-house. Young mice (aged 2-4 months) were used, given that $\mathrm{Mertk}^{-1-}$ mice have the potential to develop autoimmunity after 6 months of age (42). Arf-null ( $\left.A r f^{\prime-}\right) B C R-A B L \mathrm{p} 185^{+}$murine ALL cells expressing GFP (29) were injected via tail vein, and MRX-2843 or vehicle were administered via oral gavage beginning 1 day or 5 days after transplant. Mice with 
advanced leukemia (>20\% weight loss, tachypnea, hind-limb paralysis, minimal activity) were euthanized, and survival was monitored. Alternatively, when mice developed symptoms of leukemia (17-39 days after transplant), they and their cagemates (1 mouse per treatment group) were euthanized, and bone marrow and spleen were harvested for analysis by flow cytometry. Leukemic burden and immune cell infiltration were quantitated on an LSR II (BD Biosciences) flow cytometer and analyzed using FlowJo version X software.

Analysis of hematopoietic cells. C57BL/6 mice were treated with MRX-2843 (24) or vehicle for 24 days. Peripheral blood was collected into EDTA-coated tubes, and complete blood counts were determined using a CBC-Diff Hematology Analyzer (HESKA). To evaluate hematopoietic progenitors and immune coinhibitory receptor expression, leukocytes were isolated from spleen and/or bone marrow, stained (see below), and quantitated on a Gallios (Beckman Coulter) or LSR II (BD Biosciences) flow cytometer. Data were analyzed using FlowJo version X.

Splenocyte and leukemia cell cocultures. Splenocytes were isolated from WT and Mertk ${ }^{-1}$ C57BL/6 mice and cultured in U-bottom plates (Corning) at a 1:1 ratio with $\mathrm{Arf}^{\prime-}$ p185 B-ALL leukemia cells $\left(5.0 \times 10^{4}\right.$ cells/well) and $200 \mathrm{nM}$ MRX-2843 or DMSO vehicle for 24-72 hours. PD-L1 and PD-L2 expression on macrophages (CD11 $\left.\mathrm{b}^{+} / \mathrm{B} 220^{-}\right)$was measured using flow cytometry.

$T$ cell activation assay. U-bottom plates (Corning) were coated with anti-CD3 (10 $\mu \mathrm{g} / \mathrm{ml}$; clone 145-2C11, eBioscience) overnight at $4^{\circ} \mathrm{C}$ and then washed to remove unbound antibody. Splenocytes were isolated from WT and Mertk ${ }^{-1}$ C57BL/6 mice, and red blood cells were lysed with ACK lysing buffer (Thermo Fisher Scientific). T cells were purified by MACS magnetic cell sorting (Miltenyi Biotec) using L3T4 and Ly-2 microbeads and then cultured in anti-CD3-coated plates $\left(5 \times 10^{4}\right.$ cells/ well $)$ for 24 hours. Primed T cells were collected and cocultured with $\mathrm{Arf}^{\prime-}$ p185 B-ALL leukemia cells and whole splenocytes $\left(3.0 \times 10^{4}\right.$ T cells; $1.5 \times 10^{4}$ leukemia cells; $1.5 \times 10^{4}$ whole splenocytes) in the presence of DMSO or $200 \mathrm{nM} \mathrm{MRX-}$ 2843 for 3 days. Cells were collected and stained to detect CD4 and CD8 surface antigens, then stained using a Fixation/Permeabilization Solution Kit with GolgiPlug (BD Biosciences) to detect intracellular cytokines $(43,44)$. IFN- $\gamma$ and TNF- $\alpha$ expression on CD4 and CD8 T cells was measured by flow cytometry.

Flow cytometry antibodies. The following antibodies were used to assess hematopoietic progenitors: Sca-1 (Ly-6A/E)-FITC (clone D7), CD127-PerCP-Cy5.5 (clone A7R34), CD34-eFluor660 (clone RAM34), and CD13/32 (clone 93) from eBioscience; c-kit-PE-Cy7 (clone 2B8) from BioLegend; Flk2/ Flt3-PE (clone A2F10.1) and Lineage Antibody Cocktail V450 (including CD3e clone 500A2, CD11b clone M1/70, CD45R/B220 clone RA3-6B2, Ly-76 clone TER-119, Ly-6G/C clone RB6-8C5) from $\mathrm{BD}$ Biosciences. The following antibodies were used to assess in vivo coinhibitory receptor expression: anti-mouse CD11b-APC-CY7 (clone M1/70) and anti-mouse CD3-PE (clone 17A2) from BD Biosciences; goat anti-mouse MERTK (polyclonal) from R\&D Systems; anti-mouse CD279-BV421 (PD-1, clone 29F1A12), anti-mouse CD274-BV421 (PD-L1, clone 10F9G2), and anti-mouse CD273PE (PD-L2, clone TY25) from BioLegend; anti-mouse CD4-PE-Cy7 (clone RM4-5) and anti-mouse CD8 $\alpha$-APC-eFluor780 (clone 53-6.7) from eBioscience. The anti-mouse/rat FOXP3-APC (clone FJK16s) antibody from eBioscience was used with the FOXP3/transcription factor staining kit (eBioscience) to assess intracellular FOXP3 expression. The following anti-mouse antibodies were used for ex vivo assays: CD11b-APC (clone M1/70), B220-APC-Cy7 (clone RA3-6B2), CD273-BV421 (PDL2; clone TY25), and TNF- $\alpha-F I T C$ (clone MP6-XT22) from BD Biosciences; CD274-PECy7 (PD-L1; clone MIH5) from eBioscience; CD4-PE-Cy7 (clone GK1.5) and IFN- $\gamma-A P C$ (clone XMG1.2) from BioLegend; CD8-eFluor 450 (clone 53-6.7) from Thermo Fisher Scientific.

Statistics. Statistical analyses were performed using GraphPad Prism software (version 6.05) to compare MRX-2843- and vehicle-treated samples. Data were analyzed using 1-way ANOVA with Bonferroni's multiple-comparisons post test in experiments with 3 or more groups, or 2-tailed $t$ test in experiments with only 2 groups. Kaplan-Meier survival curves were analyzed using the Mantel-Cox test. Results were considered significant when $P$ was less than 0.05 .

Study approval. All animal experiments were conducted in accordance with relevant regulatory standards as set forth, reviewed, and approved by the IACUC of the University of Colorado and Emory University.

\section{Author contributions}

ABLS, KMJ, CJH, MGH, REP, LSP, AAH, XW, and DD designed and performed experiments and analyzed data. SVF, HSE, CTJ, and DKG analyzed data. ABLS, KMJ, CJH, HSE, DD, and DKG wrote the manuscript. 


\section{Acknowledgments}

This work was supported by the NIH (5K12HD068372, to ABLS) (R01CA137078, to DKG), the Pablove Foundation, and Federal Funds from the National Cancer Institute NCI Experimental Therapeutics (NExT) program, NIH, contract no. HHSN261200800001E. The content of this publication does not necessarily reflect the views or policies of the Department of Health and Human Services, nor does mention of trade names, commercial products, or organizations imply endorsement by the US Government. The authors thank the University of Colorado Cancer Center Flow Cytometry Core for technical assistance (P30CA046934), the Children's Healthcare of Atlanta and Emory University's Pediatric Flow Cytometry Core, and the University of Colorado Diabetes \& Endocrinology Research Center Molecular Biology Core Facility (NIH P30DK57516) for cell line authentication services.

Address correspondence to: Douglas K. Graham, Aflac Cancer and Blood Disorders Center, 2015 Uppergate Dr, Suite 404, Atlanta, Georgia 30322 Emory University School of Medicine. Phone: 404.785.3874; Email: Douglas.Graham@choa.org. Or to: Alisa Lee-Sherick, Pediatric Hematology, Oncology \& BMT, University of Colorado, School of Medicine, 12800 E 19th Ave, P18-4115, Aurora, Colorado 80045, USA. Phone: 303.724.8236; Email: Alisa.LeeSherick@ucdenver.edu.

1. Linger RM, et al. Mer receptor tyrosine kinase is a therapeutic target in pre-B-cell acute lymphoblastic leukemia. Blood. 2013;122(9):1599-1609.

2. Brandao LN, et al. Inhibition of MerTK increases chemosensitivity and decreases oncogenic potential in T-cell acute lymphoblastic leukemia. Blood Cancer J. 2013;3:e101.

3. DeRyckere D, et al. UNC2025, a MERTK small-molecule inhibitor, is therapeutically effective alone and in combination with methotrexate in leukemia models. Clin Cancer Res. 2017;23(6):1481-1492.

4. Thorp E, Cui D, Schrijvers DM, Kuriakose G, Tabas I. Mertk receptor mutation reduces efferocytosis efficiency and promotes apoptotic cell accumulation and plaque necrosis in atherosclerotic lesions of apoe ${ }^{-/-}$mice. Arterioscler Thromb Vasc Biol. 2008;28(8):1421-1428.

5. Wu Y, Singh S, Georgescu MM, Birge RB. A role for Mer tyrosine kinase in $\alpha v \beta 5$ integrin-mediated phagocytosis of apoptotic cells. J Cell Sci. 2005;118(pt 3):539-553.

6. Scott RS, et al. Phagocytosis and clearance of apoptotic cells is mediated by MER. Nature. 2001;411(6834):207-211.

7. Graham DK, DeRyckere D, Davies KD, Earp HS. The TAM family: phosphatidylserine sensing receptor tyrosine kinases gone awry in cancer. Nat Rev Cancer. 2014;14(12):769-785.

8. Rothlin CV, Ghosh S, Zuniga EI, Oldstone MB, Lemke G. TAM receptors are pleiotropic inhibitors of the innate immune response. Cell. 2007;131(6):1124-1136.

9. Zizzo G, Hilliard BA, Monestier M, Cohen PL. Efficient clearance of early apoptotic cells by human macrophages requires M2c polarization and MerTK induction. J Immunol. 2012;189(7):3508-3520.

10. Cabezón R, et al. MERTK as negative regulator of human T cell activation. J Leukoc Biol. 2015;97(4):751-760.

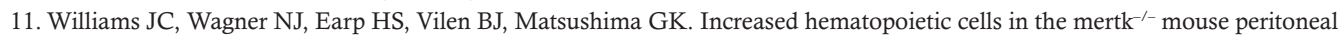
cavity: a result of augmented migration. J Immunol. 2010;184(12):6637-6648.

12. Lu Q, Lemke G. Homeostatic regulation of the immune system by receptor tyrosine kinases of the Tyro 3 family. Science. 2001;293(5528):306-311.

13. Cook RS, et al. MerTK inhibition in tumor leukocytes decreases tumor growth and metastasis. J Clin Invest. 2013;123(8):3231-3242.

14. Stanford JC, et al. Efferocytosis produces a prometastatic landscape during postpartum mammary gland involution. J Clin Invest. 2014;124(11):4737-4752.

15. Nguyen KQ, et al. Overexpression of MERTK receptor tyrosine kinase in epithelial cancer cells drives efferocytosis in a gain-offunction capacity. J Biol Chem. 2014;289(37):25737-25749.

16. Kasikara C, et al. Phosphatidylserine sensing by TAM receptors regulates AKT-dependent chemoresistance and PD-L1 expression. Mol Cancer Res. 2017;15(6):753-764.

17. Dong H, et al. Tumor-associated B7-H1 promotes T-cell apoptosis: a potential mechanism of immune evasion. Nat Med. 2002;8(8):793-800.

18. Blank C, et al. PD-L1/B7H-1 inhibits the effector phase of tumor rejection by $\mathrm{T}$ cell receptor (TCR) transgenic CD8 ${ }^{+} \mathrm{T}$ cells. Cancer Res. 2004;64(3):1140-1145.

19. Konishi J, Yamazaki K, Azuma M, Kinoshita I, Dosaka-Akita H, Nishimura M. B7-H1 expression on non-small cell lung cancer cells and its relationship with tumor-infiltrating lymphocytes and their PD-1 expression. Clin Cancer Res. 2004;10(15):5094-5100.

20. Thompson RH, Dong H, Kwon ED. Implications of B7-H1 expression in clear cell carcinoma of the kidney for prognostication and therapy. Clin Cancer Res. 2007;13(2 pt 2):709s-715s.

21. Ohigashi Y, et al. Clinical significance of programmed death-1 ligand-1 and programmed death-1 ligand-2 expression in human esophageal cancer. Clin Cancer Res. 2005;11(8):2947-2953.

22. Yamamoto R, et al. PD-1-PD-1 ligand interaction contributes to immunosuppressive microenvironment of Hodgkin lymphoma. Blood. 2008;111(6):3220-3224.

23. Wang H, et al. PD-L2 expression in colorectal cancer: Independent prognostic effect and targetability by deglycosylation. Oncoimmunology. 2017;6(7):e1327494.

24. Minson KA, et al. The MERTK/FLT3 inhibitor MRX-2843 overcomes resistance-conferring FLT3 mutations in acute myeloid 
leukemia. JCI Insight. 2016;1(3):e85630.

25. Zhang W, et al. UNC2025, a potent and orally bioavailable MER/FLT3 dual inhibitor. J Med Chem. 2014;57(16):7031-7041.

26. Linger RM, et al. Mer receptor tyrosine kinase is a therapeutic target in pre-B-cell acute lymphoblastic leukemia. Blood. 2013;122(9):1599-1609.

27. Camenisch TD, Koller BH, Earp HS, Matsushima GK. A novel receptor tyrosine kinase, Mer, inhibits TNF-alpha production and lipopolysaccharide-induced endotoxic shock. J Immunol. 1999;162(6):3498-3503.

28. Boulos N, et al. Chemotherapeutic agents circumvent emergence of dasatinib-resistant BCR-ABL kinase mutations in a precise mouse model of Philadelphia chromosome-positive acute lymphoblastic leukemia. Blood. 2011;117(13):3585-3595.

29. Williams RT, den Besten W, Sherr CJ. Cytokine-dependent imatinib resistance in mouse BCR-ABL+, Arf-null lymphoblastic leukemia. Genes Dev. 2007;21(18):2283-2287.

30. Christoph S, et al. UNC569, a novel small-molecule mer inhibitor with efficacy against acute lymphoblastic leukemia in vitro and in vivo. Mol Cancer Ther. 2013;12(11):2367-2377.

31. Lee YJ, et al. Inhibiting Mer receptor tyrosine kinase suppresses STAT1, SOCS1/3, and NF-кB activation and enhances inflammatory responses in lipopolysaccharide-induced acute lung injury. J Leukoc Biol. 2012;91(6):921-932.

32. Loke P, Allison JP. PD-L1 and PD-L2 are differentially regulated by Th1 and Th2 cells. Proc Natl Acad Sci US A. 2003;100(9):5336-5341.

33. Kostine M, et al. Increased infiltration of M2-macrophages, T-cells and PD-L1 expression in high grade leiomyosarcomas supports immunotherapeutic strategies. Oncoimmunology. 2018;7(2):e1386828.

34. Zhang Y, et al. Protein expression of programmed death 1 ligand 1 and ligand 2 independently predict poor prognosis in surgically resected lung adenocarcinoma. Onco Targets Ther. 2014;7:567-573.

35. Jung HI, et al. Overexpression of PD-L1 and PD-L2 is associated with poor prognosis in patients with hepatocellular carcinoma. Cancer Res Treat. 2017;49(1):246-254.

36. Graham DK, Bowman GW, Dawson TL, Stanford WL, Earp HS, Snodgrass HR. Cloning and developmental expression analysis of the murine c-mer tyrosine kinase. Oncogene. 1995;10(12):2349-2359.

37. Behrens EM, Gadue P, Gong SY, Garrett S, Stein PL, Cohen PL. The mer receptor tyrosine kinase: expression and function suggest a role in innate immunity. Eur J Immunol. 2003;33(8):2160-2167.

38. Levis M, Small D. FLT3: ITDoes matter in leukemia. Leukemia. 2003;17(9):1738-1752.

39. Christoph S, et al. Bioluminescence imaging of leukemia cell lines in vitro and in mouse xenografts: effects of monoclonal and polyclonal cell populations on intensity and kinetics of photon emission. J Hematol Oncol. 2013;6:10.

40. Christoph S, et al. Pre-clinical evaluation of tyrosine kinase inhibitors for treatment of acute leukemia. J Vis Exp. 2013;(79):e50720.

41. Lee-Sherick AB, et al. Efficacy of a Mer and Flt3 tyrosine kinase small molecule inhibitor, UNC1666, in acute myeloid leukemia. Oncotarget. 2015;6(9):6722-6736.

42. Cohen PL, et al. Delayed apoptotic cell clearance and lupus-like autoimmunity in mice lacking the c-mer membrane tyrosine kinase. JExp Med. 2002;196(1):135-140.

43. Henry CJ, Ornelles DA, Mitchell LM, Brzoza-Lewis KL, Hiltbold EM. IL-12 produced by dendritic cells augments CD8 ${ }^{+}$T cell activation through the production of the chemokines CCL1 and CCL17. J Immunol. 2008;181(12):8576-8584.

44. Henry CJ, et al. The roles of IL-12 and IL-23 in CD8 ${ }^{+} \mathrm{T}$ cell-mediated immunity against Listeria monocytogenes: Insights from a DC vaccination model. Cell Immunol. 2010;264(1):23-31. 